\title{
Recent progress on magnetic-field studies on quantum-spin-liquid candidates
}

\author{
Zhen Ma, ${ }^{1}$ Kejing Ran, ${ }^{1}$ Jinghui Wang, ${ }^{1}$ Song Bao, ${ }^{1}$ \\ Zhengwei Cai, ${ }^{1}$ Shichao $\mathrm{Li}^{1}{ }^{1}$ and Jinsheng Wen ${ }^{1,2, \text { * }}$ \\ ${ }^{1}$ National Laboratory of Solid State Microstructures and Department of Physics, \\ Nanjing University, Nanjing 210093, China \\ ${ }^{2}$ Collaborative Innovation Center of Advanced Microstructures, \\ Nanjing University, Nanjing 210093, China
}

\begin{abstract}
Quantum spin liquids (QSLs) represent a novel state of matter in which quantum fluctuations prevent conventional magnetic order from being established, and the spins remain disordered even at zero temperature. There have been many theoretical developments proposing various QSL states. On the other hand, experimental movement was relatively slow largely due to limitations on the candidate materials and difficulties in the measurements. In recent years, the experimental progress has been accelerated. In this topical review, we give a brief summary of experiments on the QSL candidates under magnetic fields. We arrange our discussions by two categories: i) Geometricallyfrustrated systems, including triangular-lattice compounds $\mathrm{YbMgGaO}_{4}$ and $\mathrm{YbZnGaO}_{4}, \kappa-(\mathrm{BEDT}-$ $\mathrm{TTF})_{2} \mathrm{Cu}_{2}(\mathrm{CN})_{3}$, and $\mathrm{EtMe}_{3} \mathrm{Sb}\left[\mathrm{Pd}(\mathrm{dmit})_{2}\right]_{2}$, and kagomé system $\mathrm{ZnCu}_{3}(\mathrm{OH})_{6} \mathrm{Cl}_{2}$; ii) the Kitaev material $\alpha-\mathrm{RuCl}_{3}$. Among these, we will pay special attention to $\alpha-\mathrm{RuCl}_{3}$, which has been intensively studied by our and other groups recently. We will present evidence that both supports and unsupports the QSL ground state for these materials, based on which we give several perspectives to stimulate further research activities.

PACS numbers: 61.05.fg, 75.10.Kt, 75.50.Lk
\end{abstract}

* jwen@nju.edu.cn 


\section{INTRODUCTION}

Generally, a system tends to lower its symmetry to be in the low-energy state. As a consequence, a magnetic material will break certain symmetry according to Landau's theorem, and the magnetic moments carrying by the electron spins will form an ordered pattern at low temperatures [1, 2]. Excitations associated with these ordered spins are conventional magnons with spin $S=1$ (refs [3] ). However, in systems with small spin and strong quantum fluctuations, such a conventional order can be avoided, leading to a quantum-spin"liquid" (QSL) state[10]. Now, it is known that geometrical frustration, a situation where the antiferromagnetic Heisenberg exchange interactions cannot be satisfied simultaneously among different sites in triangular [Fig. 1(a)] or kagomé lattice [Fig. 1(b)], can result in strong quantum fluctuations 11-13]. In 1973, Anderson proposed the resonant-valence-bond (RVB) model on the triangular lattice for the QSL state. It is a superposition of all possible configurations of the singlets formed by any of the two strongly interacting spins [14-17]. The elementary excitations in QSLs are fractionalized quasiparticles, e.g., charge-free $S=1 / 2$ spinons, fundamentally different from conventional magnons [14 17].

The QSL state defined by the RVB model does not have an exact solution. In 2006, Kitaev constructed an exactly-solvable $S=1 / 2$ model on the honeycomb lattice [Fig. 11(c)]. This model, as in Eq. 1, is named the Kitaev model.

$$
H=-K_{x} \sum_{x \text {-bonds }} S_{i}^{x} S_{j}^{x}-K_{y} \sum_{y \text {-bonds }} S_{i}^{y} S_{j}^{y}-K_{z} \sum_{z \text {-bonds }} S_{i}^{z} S_{j}^{z}
$$

Here, $H$ is the Hamiltonian, and $K_{x, y, z}$ are the nearest-neighbor Kitaev interactions of the $x$, $y$, and $z$ bonds on the honeycomb lattice. QSLs defined by this model is termed the Kitaev QSL. Unlike QSLs arising from geometrical frustration, the Kitaev QSL results from bonddependent interactions that frustrate spin configurations on a single site[18]. Initially, Kitaev model was treated as a toy model since the anisotropic spin interactions are unrealistic in a spin-only system. Later, it was suggested that in Mott insulators with strong spin-orbital coupling (SOC), the anisotropic Kitaev interaction may be achievable due to the spatial anisotropy of the orbitals[19]. As such, our discussions on the QSL candidates will be based on two categories: geometrical-frustration-induced ones and Kitaev QSL candidates.

Besides the rich and exotic physics of QSLs, they also hold promising application potentials, for example, quantum computation via braiding the non-Abelian anyons in these 
materials [18, 20 23]. Furthermore, understanding QSLs may help understand the mechanism of high-temperature superconductivity [24-28]. For these reasons, research on QSL has been surging in the past few decades. There have been many review articles summarizing the progress on QSLs already[11, 21, 29 42]. In this topical review, we will restrict our discussions to the measurements under magnetic fields only.
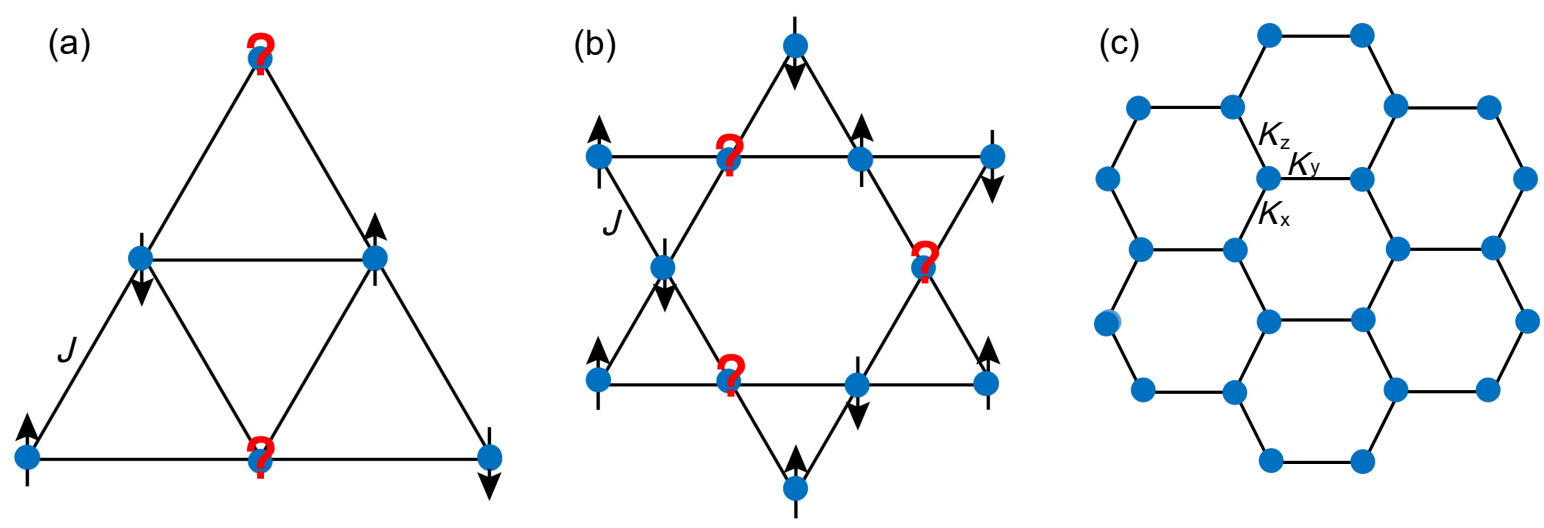

FIG. 1. Schematics of two-dimensional (a) triangular, (b) kagomé, and (c) honeycomb structures where quantum spin liquids can be realized. Arrows and question marks represent spins and geometrical frustration, respectively. $J$ is the Heisenberg exchange interaction, and $K_{x, y, z}$ are Kitaev interactions along three bonds.

In general, an external magnetic field can be detrimental to the QSL phase, as the field may induce symmetry breaking. Ultimately, when the field is strong enough, all the spins will be polarized and the moments will align with the field direction - the system is then a ferromagnet. But on the other hand, in the fully polarized state, one can extract the exchange interactions from the spin-wave excitation spectra obtained by inelastic neutron scattering (INS) measurements and understand the magnetic ground state in zero field. Investigating the magnetic excitations under fields with techniques such as nuclear magnetic resonance (NMR), muon spin relaxation ( $\mu \mathrm{SR}$ ), electron spin resonance, and terahertz spectroscopy also provides key information on the interactions underlying the exotic states of the QSL candidates. Furthermore, studying the field evolution of the thermal transport properties can also provide insights into the QSL physics. Finally, in available systems where the Kitaev physics is relevant, there are other non-Kitaev terms setting in at low temperatures, resulting in an ordered phase instead of the Kitaev QSL. In some of these 
materials, such as $\alpha-\mathrm{RuCl}_{3}$, applying a magnetic field suppresses the non-Kitaev interactions and drives the system into a possible QSL state. In this article, we will summarize the results from these magnetic-field experiments on the i) Geometrically-frustrated systems, including triangular-lattice compounds $\mathrm{YbMgGaO}_{4}$ and $\mathrm{YbZnGaO}_{4}, \kappa-(\mathrm{BEDT}-\mathrm{TTF})_{2} \mathrm{Cu}_{2}(\mathrm{CN})_{3}$, and $\mathrm{EtMe}_{3} \mathrm{Sb}\left[\mathrm{Pd}(\mathrm{dmit})_{2}\right]_{2}$, and kagomé system $\mathrm{ZnCu}_{3}(\mathrm{OH})_{6} \mathrm{Cl}_{2}$; ii) the Kitaev material $\alpha-\mathrm{RuCl}_{3}$, which has been subject to intensive investigations by our and other groups recently. We first present experimental evidence for each of these materials, then make discussions based on these results, and raise several questions in the end.

\section{GEOMETRICALLY-FRUSTRATED SYSTEMS}

\section{A. $\mathrm{YbMgGaO}_{4}$ and $\mathrm{YbZnGaO}_{4}$}

$\mathrm{YbMgGaO}_{4}$ with the quasi-two-dimensional triangular-lattice structure has been reported to be a promising QSL candidate recently 43 49]. It has a negative Curie-Weiss temperature of $\sim-4 \mathrm{~K}[43$, 44] but does not show a long-range magnetic order down to $30 \mathrm{mK}$, indicating strong geometrical frustration [45, 46]. The magnetic specific heat under different fields for $\mathrm{YbMgGaO}_{4}$ is shown in Fig. 2 (a) [43]. In zero field, there is a broad hump at $2.4 \mathrm{~K}$, instead of a sharp $\lambda$-type peak expected for a well-defined phase transition. The magnetic specific heat is large at low temperatures, suggesting a gapless ground state. By applying magnetic fields up to $9 \mathrm{~T}$, the system becomes a fully-polarized ferromagnetic state and opens a spin gap of $\sim 8.26 \mathrm{~K}$ [43, 46, 50]. $\mathrm{YbZnGaO}_{4}$, a compound isostructural to $\mathrm{YbMgGaO}_{4}$, shows very similar behaviors. In Fig. 2 (b), the magnetic specific heat results of $\mathrm{YbZnGaO}_{4}$ in zero and 9-T fields are shown. Similarly, the results indicate a gapless state in zero field, and a gapped state with a gap size of $\sim 6.18 \mathrm{~K}$ in a 9 - $\mathrm{T}$ field[51].

In zero field, the magnetic excitation spectra for both $\mathrm{YbMgGaO}_{4}$ [45, 46] and $\mathrm{YbZnGaO}_{4}$ [51] exhibit as a broad "continuum" in INS measurements, as shown in Fig. 3(a)[45]. Since QSLs are characterized by spin excitations carrying fractional quantum numbers, e.g., deconfined spinons, such observations of the continuum have been taken to be strong evidence for a QSL state in $\mathrm{YbMgGaO}_{4}[45$, 46]. Applying a magnetic field on the sample will force the magnetic moment to align with the field direction, which results in a ferromagnetic state in high fields. Paddison et al. 45] carried out INS measurements under a 7.8-T field applied 

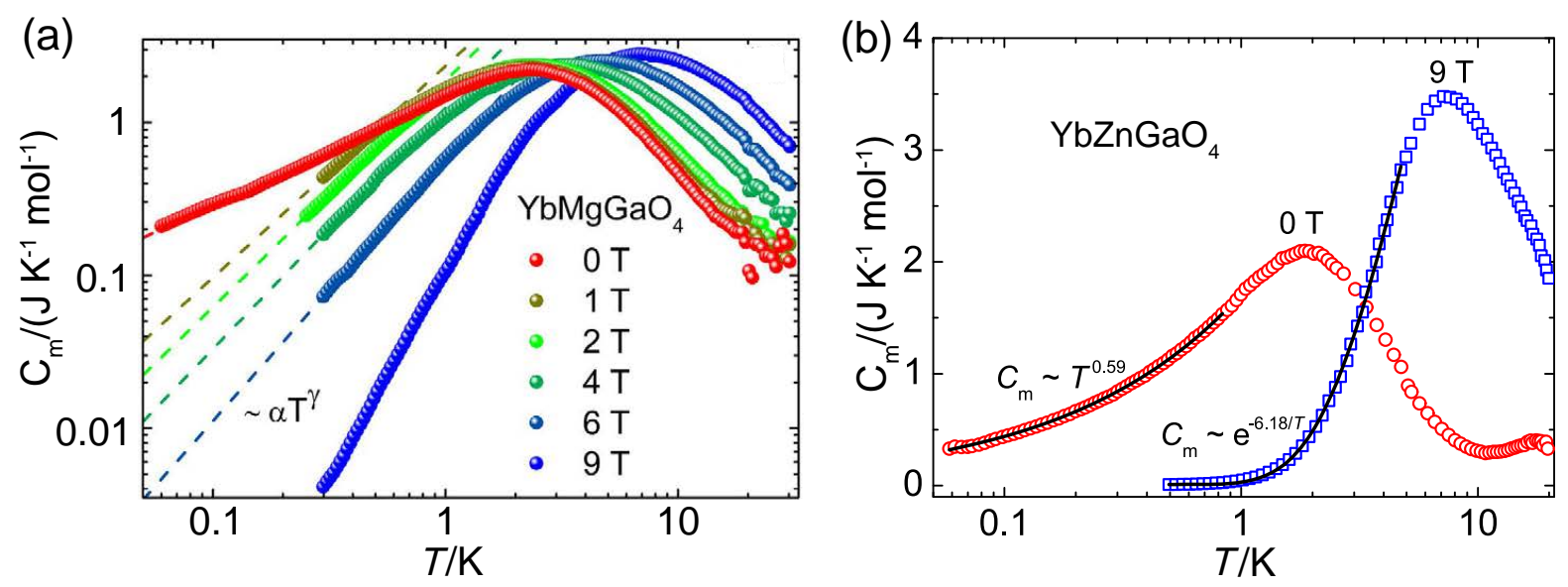

FIG. 2. (a) Temperature dependence of the magnetic specific heat of $\mathrm{YbMgGaO}_{4}$ under different magnetic fields. Dashed lines indicate power-law fits to the low-temperature data. From ref. [43]. (b) Magnetic specific heat of $\mathrm{YbZnGaO}_{4}$ measured under zero and 9-T fields. Solid lines are fits to the data. From ref. [51].

along the $c$ axis and observed the spin-wave spectra resulting from the ferromagnetic state as shown in Fig. 3 (b). In addition, there are still broad and continuum-like excitations, which are believed to be due to the disorder effect. By fitting the spectra with an anisotropic Heisenberg model plus the Zeeman term to include the field effect, they concluded that the ground state of $\mathrm{YbMgGaO}_{4}$ was a QSL in which next-nearest-neighbor exchange interactions, anisotropy, and disorder all played important roles. In $\mathrm{YbZnGaO}_{4}$, the INS results are also quite similar[51].

While results above are all consistent with $\mathrm{YbMgGaO}_{4}$ and $\mathrm{YbZnGaO}_{4}$ being QSLs, there are several issues that make this conclusion questionable: i) The small value of the exchange interaction $(\sim 0.15 \mathrm{meV})$ restricts typical INS measurements in the overdamped regime of the excitation spectra [43 46], so it is not clear whether the broad features both in momentum and energy are intrinsic or due to the large probing energy; ii) Since $\mathrm{Mg}^{2+} / \mathrm{Zn}^{2+}$ and $\mathrm{Ga}^{3+}$ in the nonmagnetic layers are randomly distributed [43, 44, 52], the disorder effect, which is considered to be detrimental to the QSL phase, is severe 445, 48, 53]; iii) Thermal conductivity $(\kappa)$ results on both $\mathrm{YbMgGaO}_{4}$ [50] and $\mathrm{YbZnGaO}_{4}$ [51] as shown in Fig. [4] strongly challenge the idea of these materials being QSLs, as we discuss in the following.

As shown in Fig. 4 , in zero field, $\kappa$ is only half of that of the nonmagnetic reference sample $\mathrm{LuMgGaO}_{4}$, in which only phonons contribute to $\kappa$. By fitting the zero-field data with 


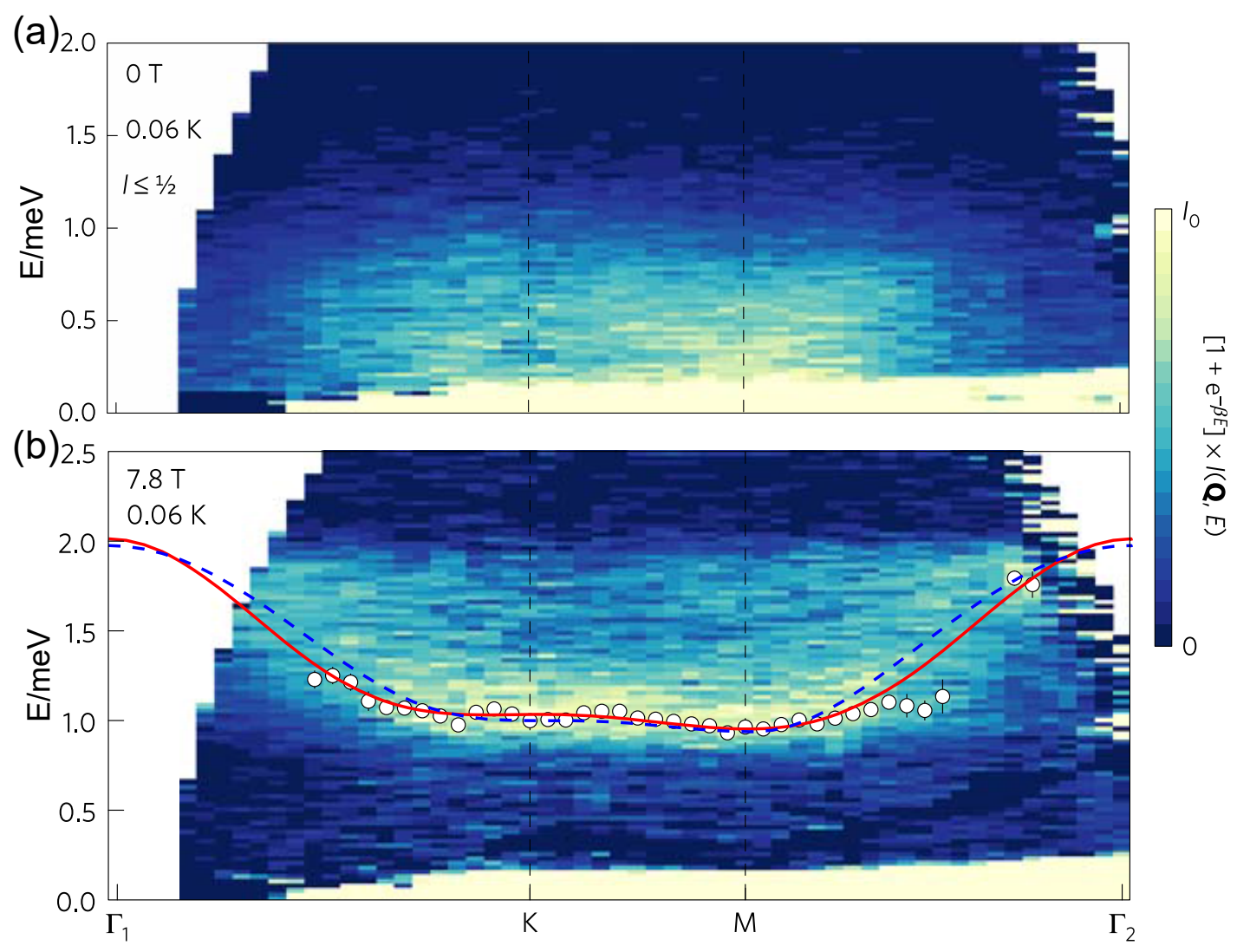

FIG. 3. Magnetic excitation spectra along high-symmetry directions under (a) zero, and (b) 7.8-T fields applied parallel to the $c$ axis. Curves are fits to the spin-wave excitations using the anisotropic spin model. From ref. [45].

$\kappa / T=\kappa_{0} / T+n T^{\beta-1}$ where $\kappa_{0}$ and $n T^{\beta}$ represent non-phonon and phonon contributions, respectively, it is shown that $\kappa_{0} / T$ for both $\mathrm{YbMgGaO}_{4}$ and $\mathrm{YbZnGaO}_{4}$ are effectively zero within experimental errors, similar to the case of $\mathrm{LuMgGaO}_{4}$ [50, 51]. Thermal conductivity can be written as $\kappa=1 / 3 C_{\mathrm{m}} v_{\mathrm{F}} l$, where $C_{\mathrm{m}}, v_{\mathrm{F}}$ and $l$ are the specific heat, Fermi velocity and the mean-free path of the quasiparticles, respectively. By assuming that the $\kappa$ at $0.1 \mathrm{~K}$ is totally contributed by the magnetic excitations, Xu et al. estimated $l$ to be $8.6 \AA$ for $\mathrm{YbMgGaO}_{4}$, only about 2.5 times of the spin-spin distance [50. In contrast, another QSL candidate $\mathrm{EtMe}_{3} \mathrm{Sb}\left[\mathrm{Pd}(d m i t)_{2}\right]_{2}$ has a high $\kappa_{0} / T=0.2 \mathrm{~W} \mathrm{~K}^{-2} \mathrm{~m}^{-1}$, which is considered to be evidence for the presence of highly mobile quasiparticles with $l$ of $\sim 1000$ times of the spin-spin distance 54 .

Furthermore, in both $\mathrm{YbMgGaO}_{4}$ and $\mathrm{YbZnGaO}_{4}$, it appears that the role of the magnetic excitations is to scatter off phonons that conduct heat. Therefore, when the magnetic 


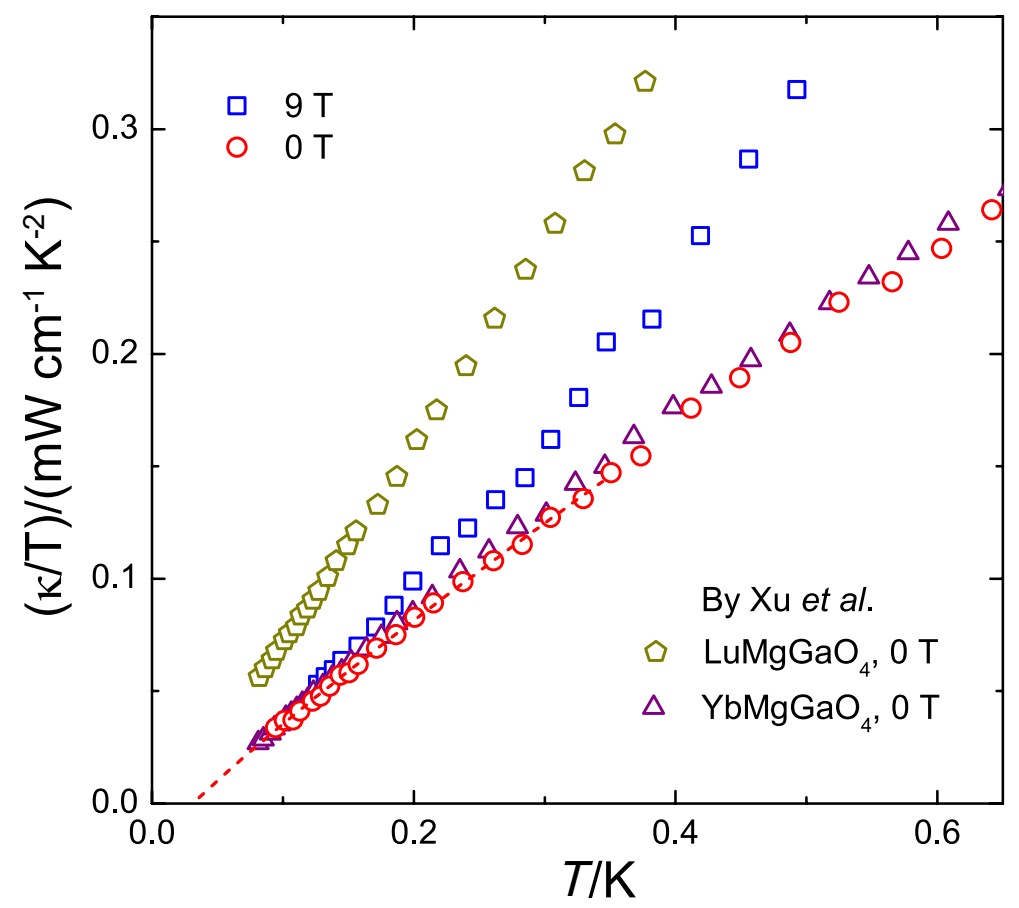

FIG. 4. The in-plane thermal conductivity of a $\mathrm{YbZnGaO}_{4}$ single crystal under zero and 9-T fields applied parallel to the $c$ axis. For comparison, the thermal conductivity of $\mathrm{YbMgGaO}_{4}$ and $\mathrm{LuMgGaO}_{4}$ reported in ref. [50] is also plotted. From ref. [51].

excitations are present, $\kappa$ is reduced - this explains the the reduction of $\kappa$ as compared to that of $\mathrm{LuMgGaO}_{4}[50,51]$. This is further manifested in the magnetic-field measurements: in a field of $9 \mathrm{~T}$ that opens a gap of 8.26 and $6.18 \mathrm{~K}$ in $\mathrm{YbMgGaO}_{4}$ [50] and $\mathrm{YbZnGaO}_{4}$ [51, respectively, there are almost no magnetic excitations to scatter phonons, so $\kappa$ increases. Therefore, a gapless QSL does not seem to be an applicable description for $\mathrm{YbMgGaO}_{4}$ and $\mathrm{YbZnGaO}_{4}$, because their significant magnetic excitations, as evidenced by the large magnetic specific heat, should contribute to $\kappa[55,56$. These results, however, can be understood within a disordered-magnet picture, in which the mean-free path of the magnons is reduced with disorder, and they are not expected to conduct heat. We will discuss it further in Sec. IV. 


\section{B. $\kappa-(\mathrm{BEDT}-\mathrm{TTF})_{2} \mathrm{Cu}_{2}(\mathrm{CN})_{3}$}

Layered organic $\kappa$-(BEDT-TTF $)_{2} \mathrm{X}$ are Mott insulators having two-dimensional triangular lattice as illustrated in Fig. 1(a). Here, BEDT-TTF (ET) denotes the electron donating molecule and $\mathrm{X}$ represents a variety of anions with closed shells [57 59]. Among these, $\kappa-(\mathrm{ET})_{2} \mathrm{Cu}_{2}(\mathrm{CN})_{3}$ is suggested to be a QSL 60 63]. The temperature dependence of magnetic susceptibility features a broad peak which can be reproduced by the triangular-lattice Heisenberg model with an exchange interaction of $J \sim 250 \mathrm{~K}[60$. The NMR spectra show neither a distinct broadening nor splitting down to $32 \mathrm{mK}$, suggesting the absence of magnetic order down to the temperature of 4 orders of magnitude lower than $J[64]$. A $\mu \mathrm{SR}$ experiment also confirms this conclusion 65$]$.

In Fig. 5(a), temperature dependences of the specific heat for $\kappa$ - $(\mathrm{ET})_{2} \mathrm{Cu}_{2}(\mathrm{CN})_{3}$ under various fields are shown together with those of other ET salts [66]. No discernible field effect is observed. From a linear extrapolation of the data in Fig. 5(a) down to zero temperature, a linearly temperature-dependent term, i.e., the electronic coefficient $\gamma$ in $C_{\mathrm{p}} T^{-1}=\gamma+\beta T^{2}$ is determined to be a finite value of $20 \pm 5 \mathrm{~mJ} \mathrm{~K}^{-2} \mathrm{~mol}^{-1}$, indicating the presence of gapless magnetic excitations in $\kappa$ - $(\mathrm{ET})_{2} \mathrm{Cu}_{2}(\mathrm{CN})_{3}$ at zero temperature [66]. This result is consistent with Anderson's proposal for the spin-liquid state in two-dimensional materials with the triangular-lattice structure[14]. For other ET salts shown in Fig. 5(a), $\gamma$ is zero as expected for nonmagnetic insulators. The $\gamma$ term is considered to be proportional to the spinon density of states, almost field independent 66].

Thermal conductivity measurements can provide key information on the elementary excitations from the magnetic ground state in a QSL candidate, as $\kappa$ is sensitive to itinerant excitations such as the spinons. In Fig. $5(\mathrm{~b}), \kappa / T$ versus $T^{2}$ for $\kappa-(\mathrm{ET})_{2} \mathrm{Cu}_{2}(\mathrm{CN})_{3}$ is shown 67]. The $\kappa / T$ has a extremely small value and tends to vanish as temperature decreases to zero. It should be stressed that the vanishing value of $\kappa / T$ at $T=0$ immediately indicates the absence of low-lying fermionic excitations [67], in sharp contrast to the specific heat data suggesting the presence of gapless excitations in ref. [66]. Moreover, the behavior under a magnetic field up to $10 \mathrm{~T}$ perpendicular to the basal plane shows a nearly parallel and small shift from that under zero field. As the zero-field data, the 10-T results also suggest the absence of magnetic excitations at low temperatures. The obvious contradiction of the specific heat and thermal conductivity results leaves the nature of the magnetic ground state 

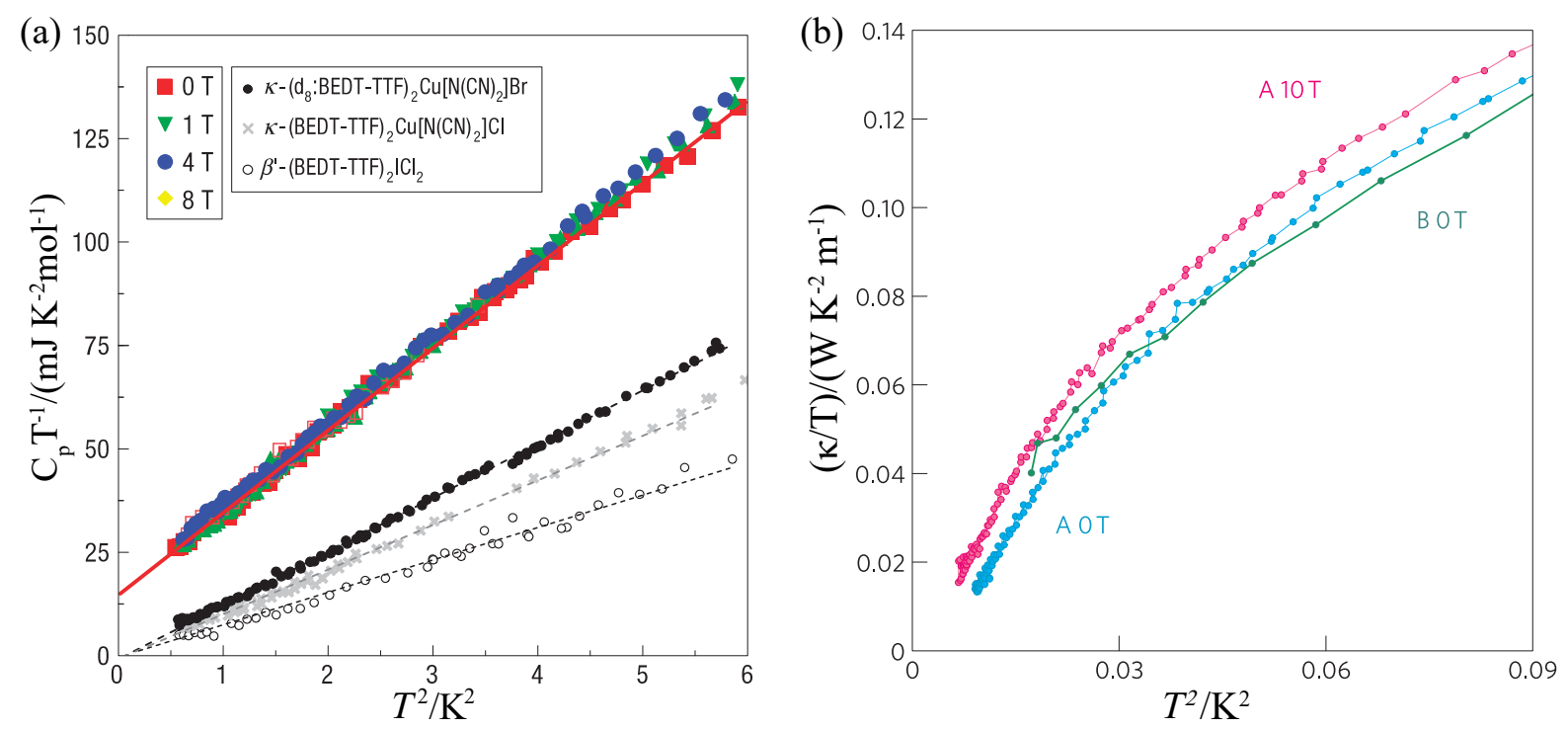

FIG. 5. (a) Specific heat of $\kappa$ - $(\mathrm{ET})_{2} \mathrm{Cu}_{2}(\mathrm{CN})_{3}$ under different fields up to $8 \mathrm{~T}$ in comparison with those of other ET-based salts. From ref. [66]. (b) Low-temperature thermal conductivity $\kappa$ of $\kappa$-(ET) ${ }_{2} \mathrm{Cu}_{2}(\mathrm{CN})_{3}$ (samples A and B). Sample A was investigated under a 10-T field applied along the $c$ axis. From ref. [67].

of $\kappa$-(ET) $)_{2} \mathrm{Cu}_{2}(\mathrm{CN})_{3}$ an open question, which will be discussed further in Sec. IV.

\section{C. $\mathbf{E t M e}_{3} \mathrm{Sb}\left[\operatorname{Pd}(\mathbf{d m i t})_{2}\right]_{2}$}

EtMe $_{3} \mathrm{Sb}\left[\mathrm{Pd}(\mathrm{dmit})_{2}\right]_{2}$ is a member of the $\mathrm{A}\left[\mathrm{Pd}(\mathrm{dmit})_{2}\right]_{2}$ family, a layered system composed of insulating $\mathrm{A}$ and conducting $\mathrm{Pd}(\mathrm{dmit})_{2}$ layers. Here, $\mathrm{A}^{+}=\mathrm{Et}_{x} \mathrm{Me}_{4-x} \mathrm{Z}^{+}\left(\mathrm{Et}=\mathrm{C}_{2} \mathrm{H}_{5}\right.$, $\mathrm{Me}=\mathrm{CH}_{3}, \mathrm{Z}=\mathrm{N}, \mathrm{P}, \mathrm{As}$, and $\left.x=0,1,2\right)$, and dmit is a 1,3-dithiole-2-thione-4,5-dithiolate 68 , 69]. Spins are on the two-dimensional triangular lattice formed by the $\mathrm{Pd}(\mathrm{dmit})_{2}$ molecules. Although the magnetic susceptibility shows a broad peak around $50 \mathrm{~K}$, no anomaly indicative of magnetic order is observed down to $2 \mathrm{~K}[29,68] .{ }^{13} \mathrm{C}$ NMR measurements down to $20 \mathrm{mK}$ also indicate the absence of long-range magnetic order at this temperature[70].

Figure $6(\mathrm{a})$ shows the specific heat of $\mathrm{EtMe}_{3} \mathrm{Sb}\left[\mathrm{Pd}(\mathrm{dmit})_{2}\right]_{2}$ in comparison with the results of $\kappa-(\mathrm{ET})_{2} \mathrm{Cu}_{2}(\mathrm{CN})_{3}[66]$ and $\mathrm{EtMe}_{3} \mathrm{P}\left[\mathrm{Pd}(\mathrm{dmit})_{2}\right]_{2}$. As in the QSL candidate $\kappa$ $(\mathrm{ET})_{2} \mathrm{Cu}_{2}(\mathrm{CN})_{3}$, there is no sharp peak indicative of long-range order over the entire temperature range measured. The specific heat of the QSL candidates $\mathrm{EtMe}_{3} \mathrm{Sb}\left[\mathrm{Pd}(\mathrm{dmit})_{2}\right]_{2}$ and $\kappa-(\mathrm{ET})_{2} \mathrm{Cu}_{2}(\mathrm{CN})_{3}$ are larger than that of $\mathrm{EtMe}_{3} \mathrm{P}\left[\mathrm{Pd}(\mathrm{dmit})_{2}\right]_{2}$ with the nonmagnetic ground 

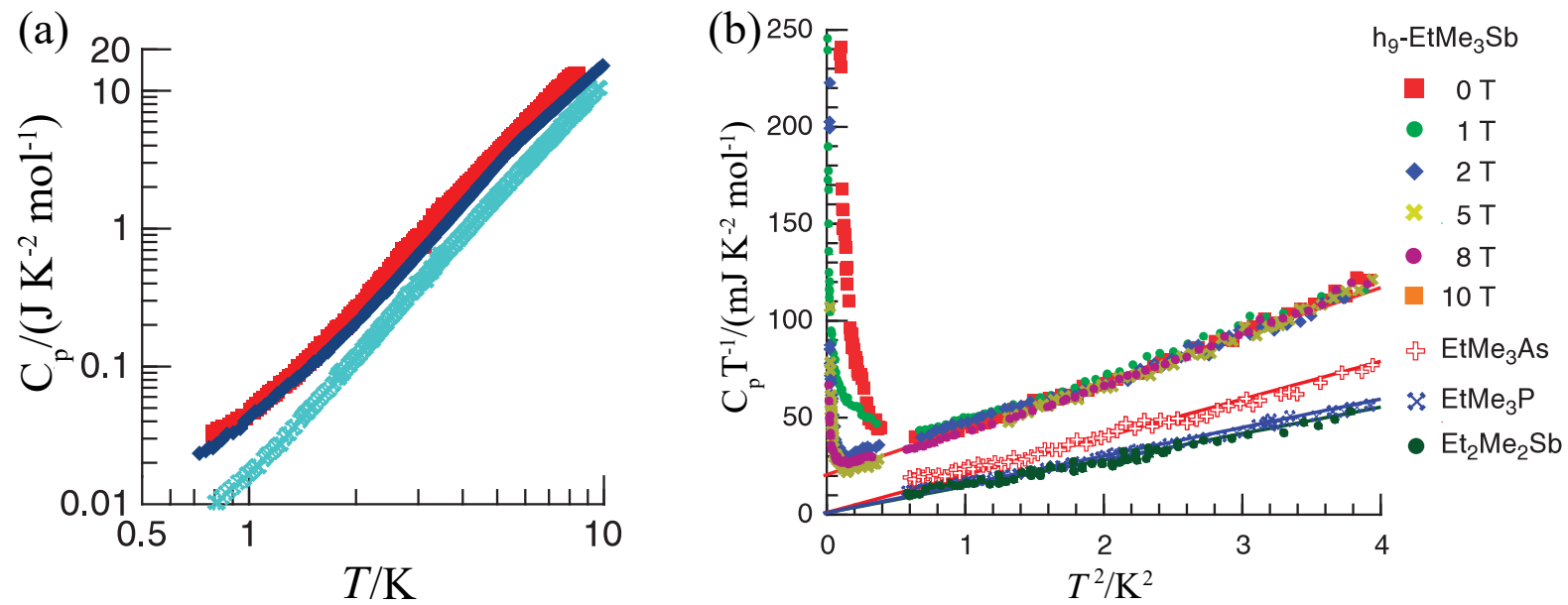

FIG. 6. (a) Specific heat data of $\mathrm{EtMe}_{3} \mathrm{Sb}\left[\mathrm{Pd}(\mathrm{dmit})_{2}\right]_{2}$ (red squares) plotted on a logarithmic scale. The data of $\mathrm{EtMe}_{3} \mathrm{P}\left[\mathrm{Pd}(\mathrm{dmit})_{2}\right]_{2}$ (cyan crosses) and $\kappa$ - $(\mathrm{ET})_{2} \mathrm{Cu}_{2}(\mathrm{CN})_{3}$ (blue diamonds) are also plotted for comparison. (b) Low-temperature specific heat of $\mathrm{EtMe}_{3} \mathrm{Sb}\left[\mathrm{Pd}(\mathrm{dmit})_{2}\right]_{2}$ under different fields up to $10 \mathrm{~T}$. The data for other nonmagnetic systems are plotted together for comparison. From ref. [71.

state of a valence-bond-solid type order occurring at $25 \mathrm{~K} 669,72,73$. This suggests that the magnetic entropy survives at low temperatures owing to the fluctuations of correlated spins in the QSL candidates[71]. The low-temperature specific heat for $\mathrm{EtMe}_{3} \mathrm{Sb}\left[\mathrm{Pd}(\mathrm{dmit})_{2}\right]_{2}$ is plotted as $C_{\mathrm{p}} T^{-1}$ vs $T^{2}$ in comparison with those of other compounds in Fig. 6(b). The finite electronic specific heat coefficient $\gamma=19.9 \mathrm{~mJ} \mathrm{~K}^{-2} \mathrm{~mol}^{-1}$ suggests the presence of gapless excitations at zero temperature[71]. The value of $\gamma$ is almost field independent, consistent with the presence of a spinon Fermi surface[71]. On the other hand, the non-QSL candidates have $\gamma$ that is effectively zero.

In Fig. 7(a), the longitudinal thermal conductivity $\kappa_{x x}$ over $T$ of $\mathrm{EtMe}_{3} \mathrm{Sb}\left[\mathrm{Pd}(\mathrm{dmit})_{2}\right]_{2}$ is plotted against $T^{2}$ in comparison with those of another QSL candidate $\kappa$ - $(\mathrm{ET})_{2} \mathrm{Cu}_{2}(\mathrm{CN})_{3}$ and a nonmagnetic compound $\mathrm{Et}_{2} \mathrm{Me}_{2} \mathrm{Sb}\left[\mathrm{Pd}(\mathrm{dmit})_{2}\right]_{2}[54]$. Interestingly, in contrast to the latter two, where $\kappa_{x x} / T$ is 0 as $T$ approaches 0 , the residual $\kappa_{x x} / T$ for $\operatorname{EtMe}_{3} \mathrm{Sb}\left[\mathrm{Pd}(\mathrm{dmit})_{2}\right]_{2}$ is $0.2 \mathrm{~W} \mathrm{~K}^{-2} \mathrm{~m}^{-1}[54]$. The presence of large residual $\kappa_{x x}$ is also confirmed in the inset of Fig. 7(a) in which $\kappa_{x x} / T$ is plotted as a function of $T$. This result is consistent with the observation of gapless magnetic excitations in the specific heat measurement. By using $\kappa_{x x}=1 / 3 C_{\mathrm{m}} v_{\mathrm{F}} l$, it is suggested that there are highly mobilized magnetic excitations with 

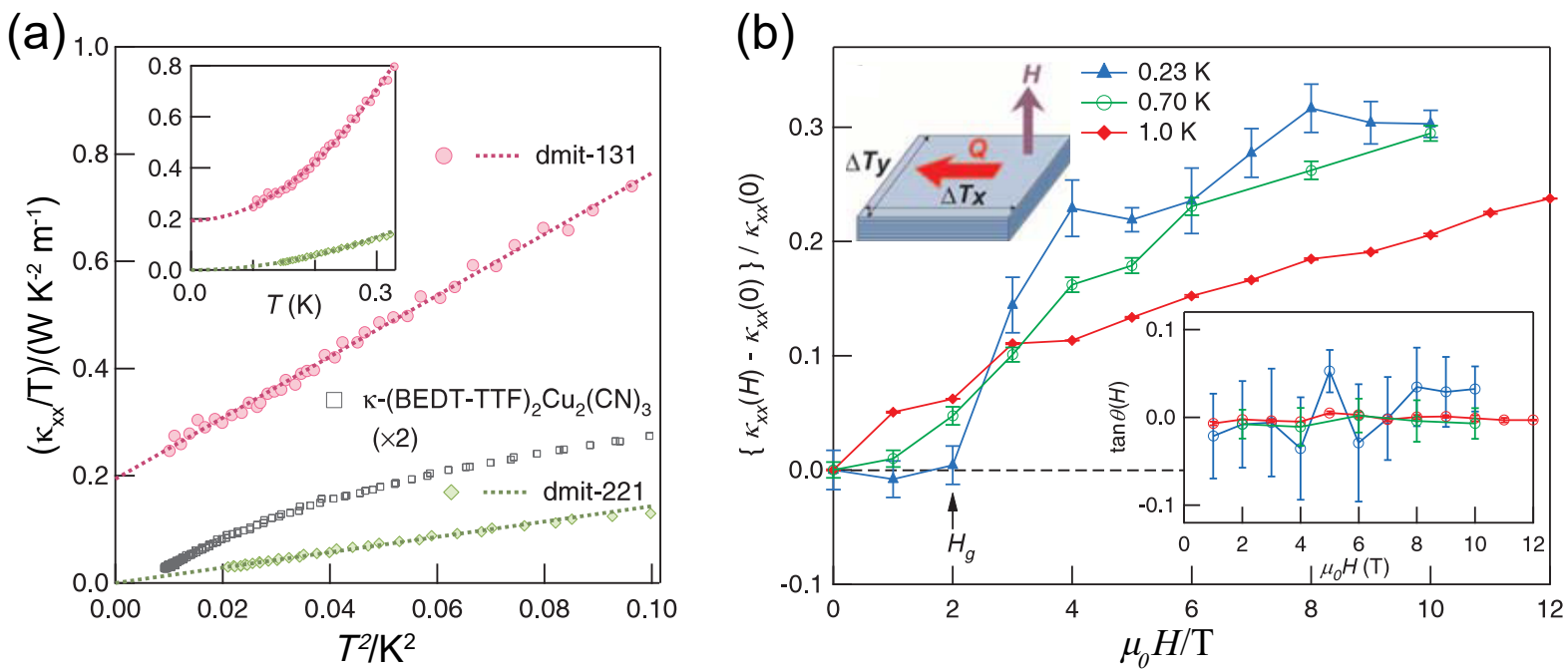

FIG. 7. (a) Low-temperature plot of $\kappa_{x x} / T$ as a function of $T^{2}$ of dmit-131 (circles), dmit-221 (diamonds), and $\kappa$-(ET) $)_{2} \mathrm{Cu}_{2}(\mathrm{CN})_{3}$ (squares). The inset shows $\kappa / T$ plotted as a function of $T$. (b) Field dependence of $\kappa$ normalized by the zero-field value, $\left[\kappa_{x x}(H)-\kappa_{x x}(0)\right] / \kappa_{x x}(0)$ of dmit-131 at low temperatures. Upper left inset shows a schematic of the measurement setup. The lower left inset shows thermal-Hall angle $\tan \theta(H)=\kappa_{x y} /\left(\kappa_{x x}-\kappa_{x x}^{\mathrm{ph}}\right)$ as a function of the field value $\mu_{0} H$ at $0.23 \mathrm{~K}$ (triangles), $0.70 \mathrm{~K}$ (circles), and $1.0 \mathrm{~K}$ (diamonds). $\kappa_{x y}$ and $\kappa_{x x}^{\mathrm{ph}}$ are the thermal-Hall conductivity and phonon-contributed thermal conductivity, respectively. From ref. [54].

a mean-free path $l$ of $\sim 1000$ times of the spin-spin distance [54]. Remarkably, this is a rare example where thermal conductivity measurements show a finite $\kappa_{x x} / T$ at zero temperature. The results indicate the presence of gapless magnetic excitations consistent with the specific heat data[71].

The magnetic-field dependence of $\kappa_{x x}$ is shown in Fig. 77(b) [54]. At the lowest temperature, $\kappa_{x x}(H)$ under low fields is insensitive to the magnetic field strength $\mu_{0} H$ but displays a steep increase above a characteristic magnetic field of $2 \mathrm{~T}$. This behavior is less profound with increasing temperature. At $1 \mathrm{~K}, \kappa_{x x}$ becomes linear. The observed field dependence is interpreted as the presence of spin-gap-like excitations at low temperatures, along with the gapless excitations inferred from the residual $\kappa_{x x} / T$ and finite $\gamma[54$, 71]. The gap behavior is also suggested from the zero-field thermal conductivity measurement where $\kappa_{x x} / T$ vs. $T$ shows a broad peak at $\sim 1 \mathrm{~K}[54$. 


\section{D. $\mathrm{ZnCu}_{3}(\mathrm{OH})_{6} \mathrm{Cl}_{2}$}

$\mathrm{ZnCu}_{3}(\mathrm{OH})_{6} \mathrm{Cl}_{2}$ known as Herbertsmithite has a three-dimensional rhombohedral structure and consists of two-dimensional kagomé-lattice planes of spin- $1 / 2 \mathrm{Cu}^{2+}$ ions separated by nonmagnetic layers formed by $\mathrm{Zn}^{2+}[74]$. Geometrical frustration on such a structurally perfect kagomé lattice is expected to be strong and many interesting phenomena may emerge [35, 75]. Because large-size single crystals are available for this material[76], it has been heavily studied by various experimental techniques. Previous measurement results 77 79] of polycrystalline Herbertsmithite suggest that there is no static magnetic order nor spin freezing down to $50 \mathrm{mK}$ despite the large Curie-Weiss temperature of $-314 \mathrm{~K}$ [74]. Specific heat shows no sharp $\lambda$-type peak down to $\sim 100 \mathrm{mK}[77]$. When applying a magnetic field to the sample, the specific heat is changed rapidly as shown in Fig. 8 (ref. [77]). Under a 14-T field, the low-temperature specific heat is largely reduced, because magnetic excitations are gapped, leaving only phonons contributing to the specific heat. On the other hand, at high temperatures where phonons dominate the specific heat, there is hardly any field effect.

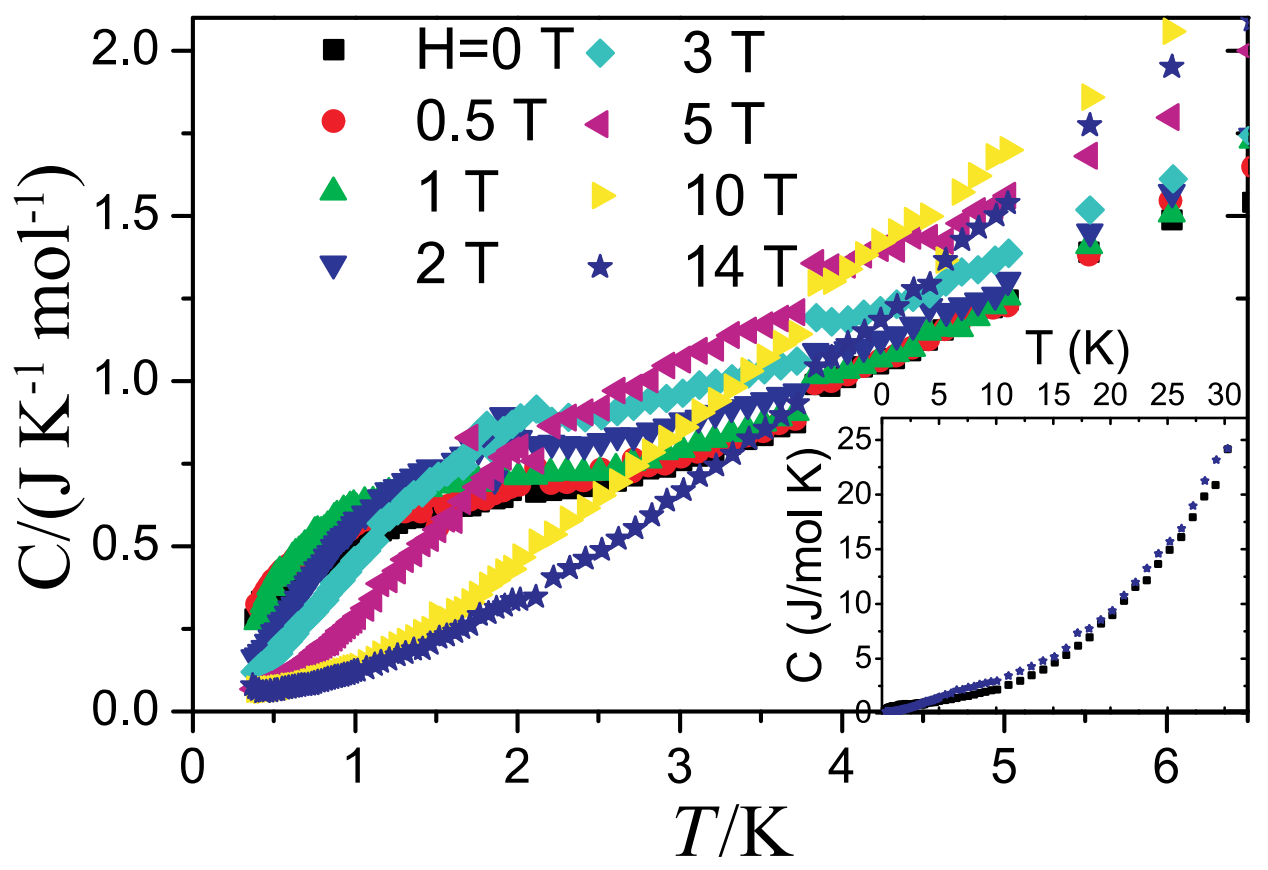

FIG. 8. Specific heat of Herbertsmithite measured under several different magnetic fields up to 14 T. Inset: Specific heat plotted over a wider temperature range under zero (squares) and 14-T (stars) fields. From ref. [77]. 

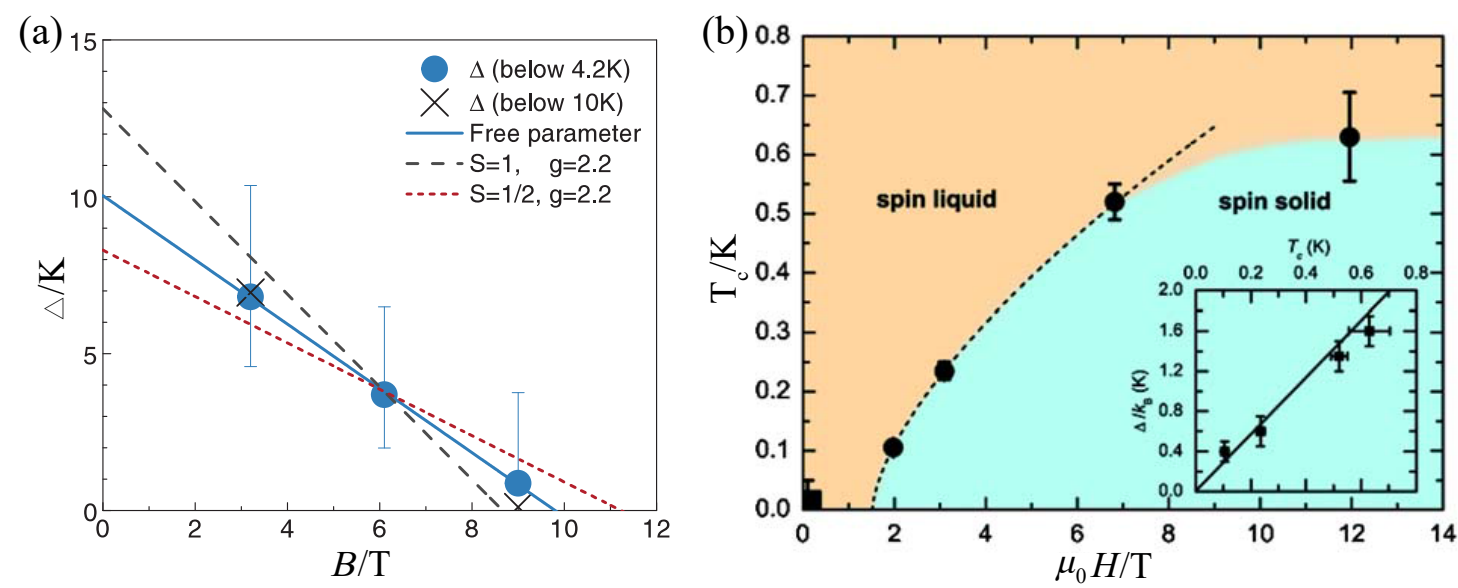

FIG. 9. (a) Spin excitation gap $\Delta$ of Herbertsmithite obtained by fitting NMR spectra with a temperature range up to $4.2 \mathrm{~K}$ (circles) and $10 \mathrm{~K}$ (crosses). Dashed and dotted lines represent the best fits under the constraint of $S=1$ and $1 / 2$, respectively. The solid line represents the best free-parameter fit. From ref. [80]. (b) Transition temperature $T_{c}$ versus magnetic field phase diagram of Herbertsmithite. The inset plots $\Delta$ as a function of $T_{c}$. From ref. [81].

The availability of large single crystals of Herbertsmithite[76] makes it possible to carry out INS measurements, which reveal a broad continuum expected for a QSL state[82]. The INS results show no spin gap down to $0.25 \mathrm{meV}$. However, previous calculation results by density matrix renormalizaton group indicate that the ground state of the Heisenberg model on a kagomé lattice is a fully gapped QSL[83]. To understand the magnetic ground state of Herbertsmithite, Asaba et al.[84] performed magnetization measurements on a single crystal using torque magnetometry with an intense magnetic field up to $31 \mathrm{~T}$. Based on the observation that the effective magnetic susceptibility under high fields is independent of the temperature within the low-temperature range between $20 \mathrm{mK}$ and $5 \mathrm{~K}$, they considered the ground state to be gapless, consistent with the INS results [82].

However, as shown in Fig. 9(a), NMR measurements on single crystals of Herbertsmithite reveal a finite gap value $0.86 \pm 0.26 \mathrm{meV}$ by extrapolating the fitted values of gap to zero field [80]. This value is close to a later INS measurement which identifies a spin gap of $0.7 \mathrm{meV}$ by modeling the momentum-integrated data as the sum of the contributions from a damped harmonic oscillator and the gapped excitations[85]. We note that a more recent calculation employing the tensor network shows that the ground state is a gapless QSL[86]. Concerning the nature of the ground state for this kagomé compound, it turns out that no 
consensus has been reached so far.

Moreover, NMR measurements on polycrystalline samples identify a quantum critical point at a critical field $B_{c}$ of $1.53 \mathrm{~T}$, at which the system is believed to evolve from a spin liquid to a solid, as illustrated in Fig. 9(b) [81]. The high-field, low-temperature spin-solid state is featured by frozen spins with slow relaxations. As shown in the inset of Fig. 9(b), the gap size in the spin-solid state is roughly proportional to the critical temperature $T_{c}[81]$.

\section{KITAEV MATERIALS- $-\alpha-\mathrm{RuCl}_{3}$}

Different from aforementioned QSL candidates which have either triangular or kagomé lattice where antiferromagnetic exchange interactions are geometrically frustrated, the Kitaev QSL has honeycomb lattice where the frustration on a single site arises from the bonddependent spin anisotropy [18]. Possible realization of such an exotic state has been suggested in SOC-assisted Mott insulators such as $\mathrm{Na}_{2} \mathrm{IrO}_{3}$ [19, 42, 87 91], and $\mathrm{Li}_{2} \mathrm{IrO}_{3}$ [91 95]. These materials have the honeycomb lattice as shown in Fig. 1(c). Due to the combination of the cubic crystal electric field, strong SOC, and electronic correlations, the ground state is Krammers doublets with an effective spin of 1/2 (refs [96 98]). Furthermore, because of the strong SOC, the effective spin is expected to be anisotropic due to the spatial anisotropy of the $5 d$ orbitals of $\mathrm{Ir}^{4+}$. Therefore, the bond-dependent anisotropic Kitaev interaction may be realized on the honeycomb lattice[18]. However, it is found that they are not QSLs but are instead magnetically ordered at low temperatures [42, 91 95, 98 101]. Nevertheless, subsequent experimental and theoretical works suggest that the magnetic orders in these materials are unconventional, signifying the presence of notable Kitaev interaction. For instance, it is suggested that the zigzag magnetic order in $\mathrm{Na}_{2} \mathrm{IrO}_{3}$ can be understood within a Heisenberg-Kitaev model[89, 102]. In the phase diagram constructed using this model, there are regions where the Heisenberg interaction is small and the Kitaev interaction is

dominant, leading to the Kitaev QSL phase. Because of this, finding Kitaev QSLs in related materials is still encouraging. In the following, we will discuss another Kitaev material $\alpha$ $\mathrm{RuCl}_{3}$, which has been the focus of recent research due to the availability of high-quality single crystals and feasibility of neutron scattering measurements.

$\alpha-\mathrm{RuCl}_{3}$ has two-dimensional honeycomb layers formed by the $4 d \mathrm{Ru}^{3+}$ ions $\left.103-108\right]$. In fact, realization of the Kitaev interaction in materials with $4 d$ electrons does not sound 
very promising in the beginning, because their SOCs are smaller compared to those of the $5 d$ systems. However, although the absolute value of the $\mathrm{SOC}$ in $\mathrm{RuCl}_{3}$ is smaller, the almost- $90^{\circ}$ bond angles of the $\mathrm{Cl}-\mathrm{Ru}-\mathrm{Cl}$ bonds of the edge-shared $\mathrm{RuCl}_{6}$ octahedra makes the cubic crystal electric field win and the SOC become a dominant effect[103 112]. Thus, similar to iridates, $\alpha-\mathrm{RuCl}_{3}$ is also an SOC-assisted Mott insulator with an effective spin of $1 / 2$, and the strong spatial anisotropy of the $4 d$ orbitals combined with the SOC makes the bond-dependent Kitaev interaction significant[18, 103, 104, 113]. However, similar to $\mathrm{Na}_{2} \mathrm{IrO}_{3}$, the ground state of $\alpha-\mathrm{RuCl}_{3}$ is not a Kitaev QSL, but a zigzag magnetic order state instead[103 108]. It has been proposed that the zigzag order is an indication for the presence of the Kitaev interaction in this system[19, 87, 105 108. Moreover, INS results indicate that the ground state of $\alpha-\mathrm{RuCl}_{3}$ may be proximate to the Kitaev QSL phase[114], and both INS[114 117] and Ramman studies[118, 119] observe broad continuous magnetic excitations that can be associated with fractionalized excitations resulting from the Kitaev QSL phase.

By analyzing the INS spectra, magnetic interactions governing the ground state can be extracted. In Fig. 10(a), we show the spin-wave excitation spectra resulting from the zigzag order state[115]. Instead of using the widely considered Heisenberg-Kitaev $(H-K)$ model[19, 87] to fit the spectra, a $K$ - $\Gamma$ effective-spin model is used, where $K$ and $\Gamma$ represent Kitaev and off-diagonal exchange interactions, respectively. This minimal model describing the ground state of $\alpha-\mathrm{RuCl}_{3}$ is first proposed by Wang et al. in ref. [120]. They recognize the importance of the off-diagonal interactions, but on the other hand, they find that the Heisenberg interactions are at least an order of magnitude smaller than either $K$ or $\Gamma$ in the parameter range relevant to this material. Fits to the INS spectra with this model yield a ferromagnetic $K$ of $-6.8 \mathrm{meV}$ and a $\Gamma$ of $9.5 \mathrm{meV}[115]$, very close to the calculated results of $K=-5.5 \mathrm{meV}$ and $\Gamma=7.6 \mathrm{meV}$ reported in ref. [120]. These results unambiguously demonstrate that the Kitaev interaction is large and exists in real material.

As mentioned above, the ground state of $\alpha-\mathrm{RuCl}_{3}$ is the zigzag order state instead of a Kitaev QSL. Nevertheless, the magnetic order is rather fragile, with an ordered moment of $\sim 0.4 \mu_{B}$ and an ordering temperature of $\sim 8 \mathrm{~K}[103-108$, 115]. Such a fragile order can be fully suppressed by either an in-plane magnetic field[106, 121, 122] or pressure[123]. How do the magnetic excitations behave in the high-field state? Is the high-field disordered state a QSL? If the high-field state is a QSL, what is the relationship between this phase 

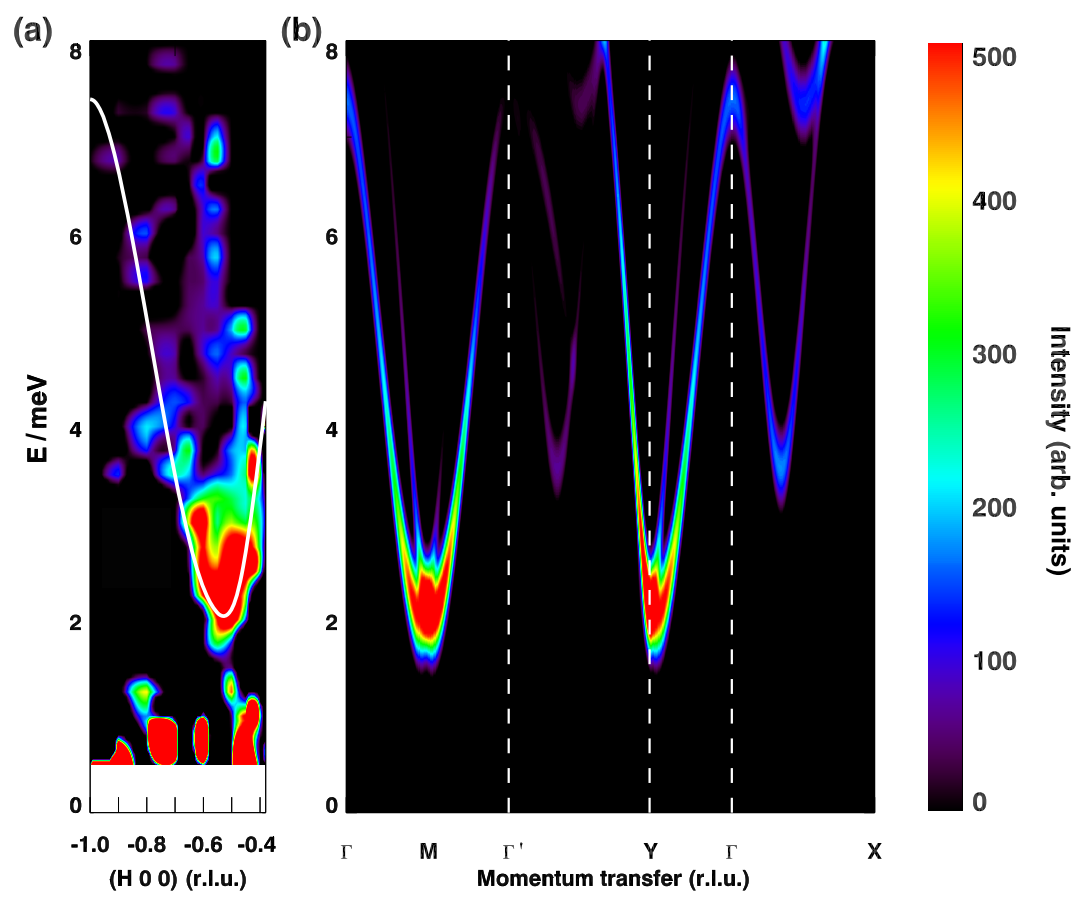

FIG. 10. (a) Magnetic dispersion along the [100] direction obtained by INS experiments. The solid line is the calculated dispersion as shown in (b). (b) Calculated spin-wave spectra along high-symmetry paths. From ref. [115].

and the long-sought Kitaev QSL? To answer these questions, measurements utilizing various experimental techniques have been carried out [106, 121, 122, 124 133]. Some of these results are discussed as following.

By following the magnetic-field dependence of the magnetization and specific heat, it is found that the zigzag order is gradually suppressed, and the system becomes a magnetically disordered state at $\sim 7.5 \mathrm{~T}[124$, consistent with earlier reports on the field effect[106, 121, 122. NMR spectra on high-quality single crystals also indicate that there is a quantum critical point at $B_{c} \sim 7.5 \mathrm{~T}[124]$. Above $B_{c}$, the spin-lattice relaxation rate $1 / T_{1}$ of ${ }^{35} \mathrm{Cl}$ shows a power-law behavior as $1 / T_{1} \sim T^{\alpha}$. In a field range between 8 and $16 \mathrm{~T}, \alpha \approx 3$, suggesting a field-induced QSL featuring Dirac nodal-like spin excitations. A phase diagram summarizing these results is shown in Fig. 11(a). Intensive research on the high-field state utilizing various techniques such as magnetization[106, 121, 122, 124, 132, 135], specific heat [106, 124126, 134, 135], magnetodielectric[128, neutron diffraction[122, 126, 132, NMR[124, 134], magnetic torque [127, thermal conductivity [127, 133, 135], terahertz spectroscopy [129, 130], 
(a)

(d)
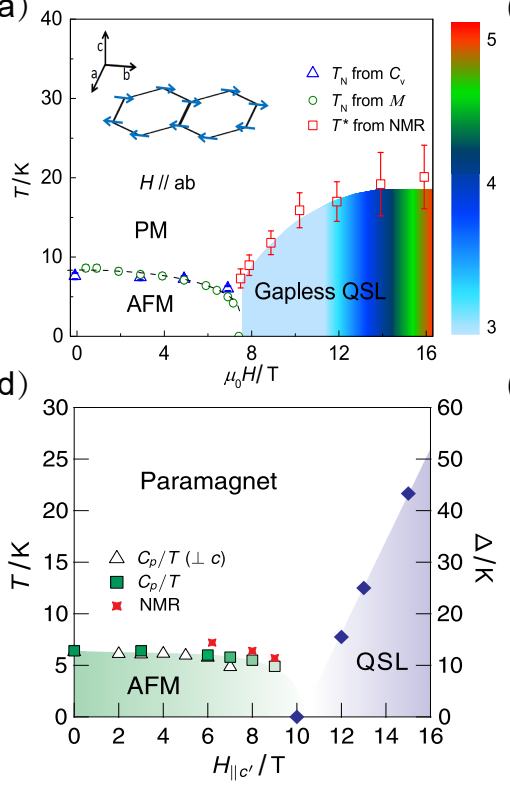

(b)

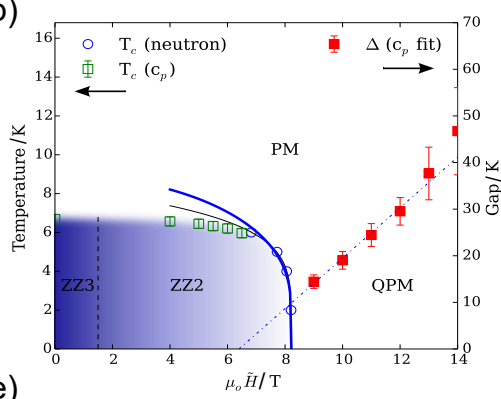

(e)

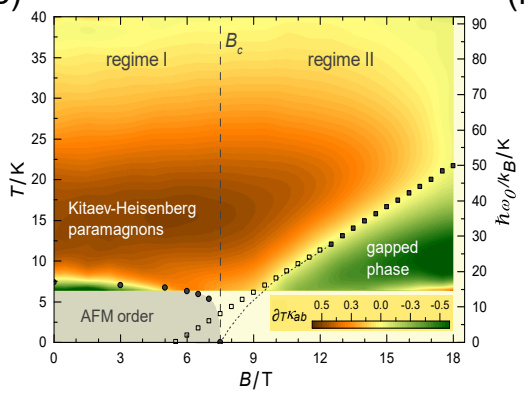

(c)

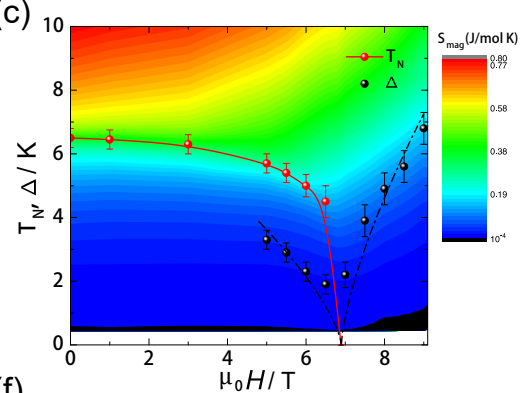

(f)

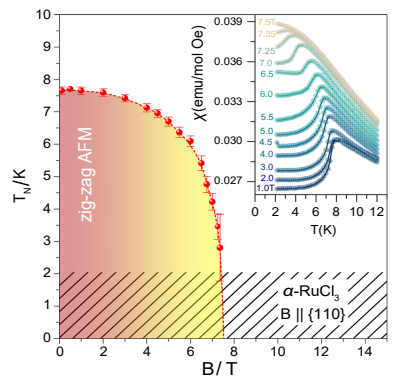

FIG. 11. Magnetic phase diagram of $\alpha-\mathrm{RuCl}_{3}$ obtained from various measurements. (a) PM and AFM represent paramagnet and zigzag order, respectively. Low-field region of the phase diagram is constructed using magnetization and specific heat data, and the high-field region is using NMR data. The magnetic field is applied in the $a-b$ plane. The contour map indicates the exponent of the temperature dependence of the spin-lattice relaxation rate. The inset illustrates the zigzag order in the low-field state. From ref. [124]. (b) ZZ and QPM represent zigzag order and quantum paramagnet, respectively. The phase boundary between ZZ2 and PM is the transition temperature obtained from specific heat and neutron diffraction measurements. The thick solid line is a fit with the transverse-field Ising model, and the thin solid line is a power-law fit. The dashed line is a power-law fit to the gap size $\Delta$. From ref. [126] (c) Transition temperatures and gap values obtained from specific heat measurements. The solid line is a guide to the eye. Dashed lines are the fits of the gap function. The magnetic entropy is shown in a color scale. From ref. [125]. (d) Phase diagram obtained from specific heat and NMR measurements along with the field dependence of the spin gap $\Delta$ extracted from the nuclear-spin relaxation rate (right axis). From ref. [134]. (e) False-color representation of the $T$ derivative of the $a b$-plane thermal conductivity $\left(\kappa_{a b}\right)$ together with the gap values (solid squares) extracted from the phononic fits. The color scale is in the unit of $\mathrm{W} / \mathrm{K}^{2} \mathrm{~m}$. From ref. [133]. (f) Magnetic transition temperature as a function of field obtained from susceptibility measurements with field applied along the [110] direction. The inset shows the susceptibility data. From ref. [132]. 
and electron spin resonance[131, has resulted in many somewhat similar phase diagrams, some of which are shown in Fig. 11.
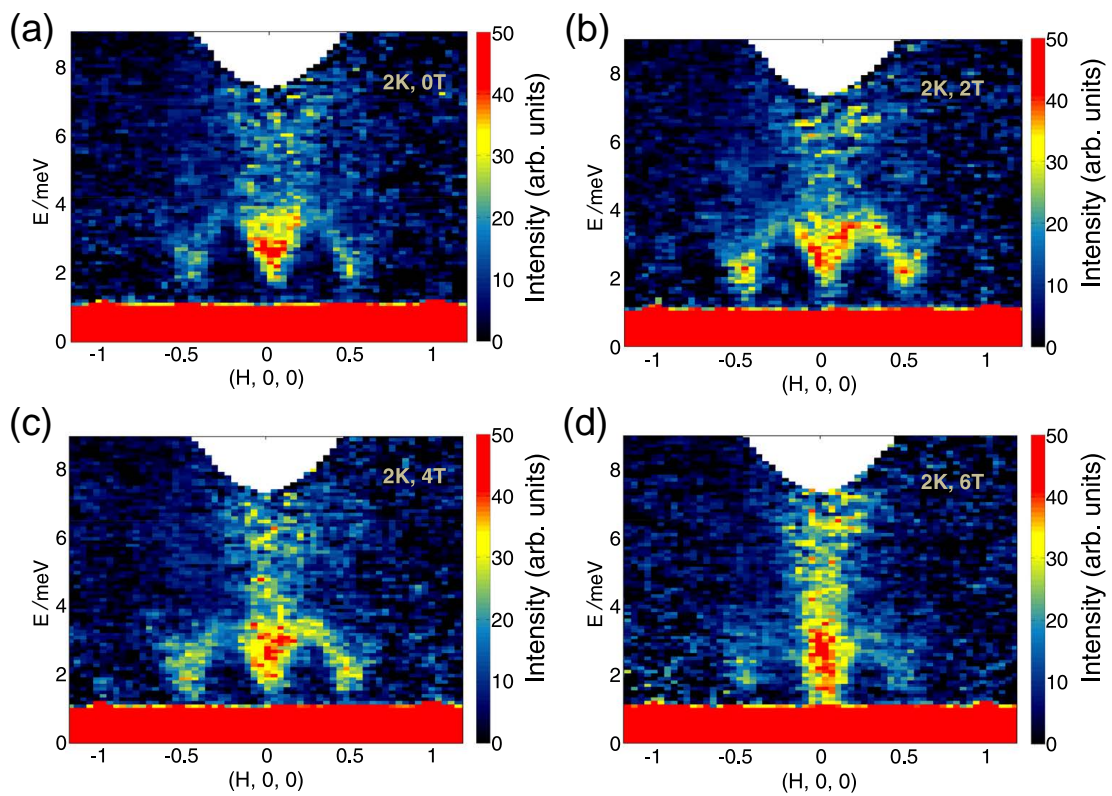

(d)
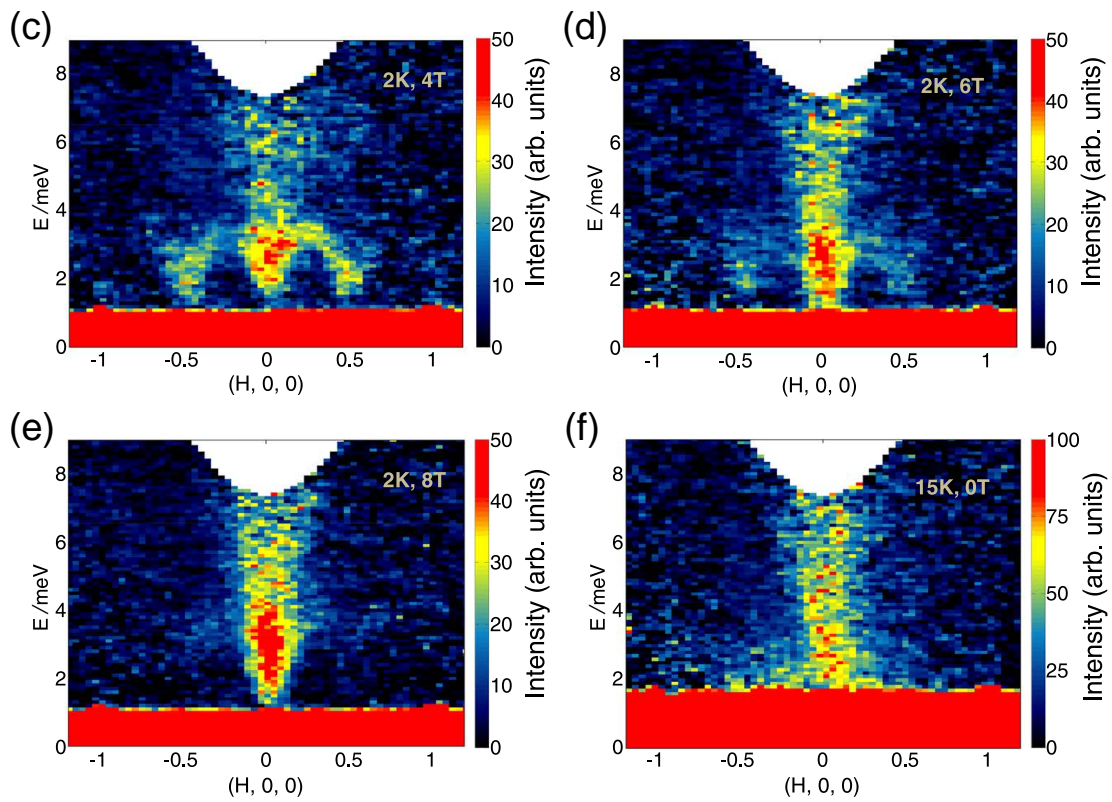

FIG. 12. (a)-(e) Magnetic-field evolution of the INS spectra along the [100] direction, measured at $T=2 \mathrm{~K}$ with an external magnetic field applied in the $a-b$ plane. (f) The zero-field data at $15 \mathrm{~K}$, above the ordering temperature. From ref. [132].

On one hand, these phase diagrams all show that the zigzag magnetic order is gradually suppressed by an in-plane magnetic field, and the system reaches a quantum critical point around $B_{c} \approx 7.5 \mathrm{~T}$. Furthermore, accumulating evidence suggests that the high-field disordered state above $B_{c}$ is a QSL. In particular, Banerjee et al. have carried out INS measurements to examine the magnetic-field evolution of the magnetic excitations, and some of the results are shown in Fig. 12 132]. They find that the spin-wave excitations associated with the zigzag order near the M point is suppressed with the field. As shown in Fig. 12 (e), above $B_{c}$, at $\mu_{0} H=8 \mathrm{~T}$, excitations near the $\mathrm{M}$ point are completely gone, and only exci- 
tations near the $\Gamma$ point remain, similar to the results under zero field above the ordering temperature, as shown in Fig. 12 (f). By comparing with calculations, they suggest that the excitations under high fields resemble those of a Kitaev QSL[132].
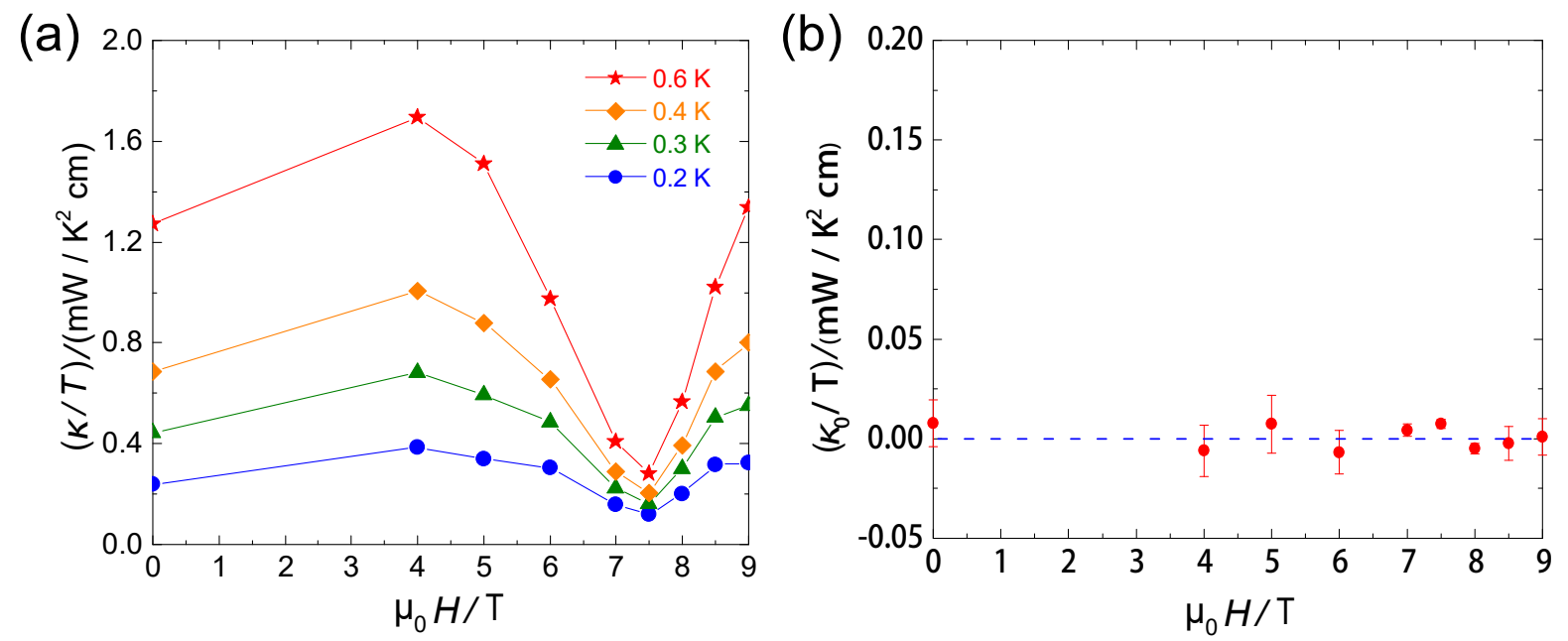

FIG. 13. (a) Field dependence of $\kappa / T$ at various temperatures. The minimum of $\kappa / T$ at $\sim 7.5 \mathrm{~T}$ corresponds to $B_{c}$, where the zigzag magnetic order disappears. (b) Field dependence of the residual linear term $\kappa_{0} / T$. From ref. [135].

On the other hand, the nature of the field-induced QSL phase, in particular, whether the low-energy magnetic excitations associated with this state are finite [124, 127] or fully gapped[125, 126, 130 132, 134, is still under debate. To resolve this issue, Yu et al. [135] have performed ultralow-temperature thermal conductivity measurements down to $80 \mathrm{mK}$ under magnetic fields, and the results are summarized in Fig. 13 . As shown in Fig. 13 (a), $\kappa / T$ increases with field and then decreases to a minimum at the critical field $B_{c}$. Above $B_{c}, \kappa / T$ increases with field again. These results clearly show that there is a quantum critical point at $B_{c}$, consistent with other works [124-127, 130, 131, 134]. By examining the residual $\kappa$ at zero temperature, it is found that $\kappa_{0}$ is effectively zero in the whole field range probed [Fig. 13(b)]. In the low-field range below $B_{c}$, there is a spin-anisotropy gap about $2 \mathrm{meV}$ associated with the zigzag order[114, 115, 132]. Therefore, absence of the thermal conductivity at zero temperature is expected. If the high-field state is also fully gapped as suggested in refs [125, 126, 130 132, 134], these thermal conductivity results are easily explainable. However, as shown in Fig. 11(a), a gapless QSL state near $B_{c}$ is suggested[124]. We here provide one possible solution to reconcile this discrepancy. According to the report, this gapless state 
is in fact featured by Dirac-like excitations with gap nodes in the momentum space[124]. In this case, the magnetic density of states, represented as $C_{\mathrm{m}}$ in $\kappa=1 / 3 C_{\mathrm{m}} v_{\mathrm{F}} l$ is small. At present, the estimated magnetic specific heat $C_{\mathrm{m}}$ has big uncertainties due to the lack of proper reference sample to subtract the phonon contributions [106, 124, 126, 134, 135]. If the real $C_{\mathrm{m}}$ is small, then the gapless state with nodal excitations proposed in ref. [124] can also be consistent with the thermal conductivity results[135].

\section{DISCUSSIONS}

As partially reflected from the discussions above, research on the QSL candidates has been quite dynamics. A lot of progress has been made already in recent years. However, it still lacks an ideal QSL candidate so far. Quite often, the spin- "liquid" behavior may have some other origins than quantum fluctuations. Below, we will show some examples.

There is accumulating evidence suggesting $\mathrm{YbMgGaO}_{4}$ to be a promising candidate as a gapless QSL[43 49]. However, the report of no positive contributions from the magnetic excitations to the thermal conductivity is difficult to be reconciled with the gapless QSL picture [50]. One possibility is that the severe disorder effect caused by the random mixing of $\mathrm{Mg}^{2+}$ and $\mathrm{Ga}^{3+}$ makes the otherwise itinerant spinons localized and thus not conduct heat [43, 44, 48, 50, 52]. However, the disorder is considered to be detrimental to the QSL phase for this compound[53]. Ma et al. [51] have carried out measurements on $\mathrm{YbZnGaO}_{4}$, a sister compound of $\mathrm{YbMgGaO}_{4}$, utilizing various techniques, including d.c. susceptibility, specific heat, INS, and ultralow-temperature thermal conductivity. They have found that a spin-glass phase can explain the experimental observations in $\mathrm{YbZnGaO}_{4}$ : including no longrange magnetic order, prominent broad excitation continua observed by INS, and absence of magnetic thermal conductivity. By analogy, they suggest the spin-glass phase to be also applicable to $\mathrm{YbMgGaO}_{4}$.

The spin-glass phase, with frozen, short-range correlations below the freezing temperature $T_{\mathrm{f}}[136$ 138], can be identified from the a.c. susceptibility. Ma et al. have performed such measurements on both $\mathrm{YbMgGaO}_{4}$ and $\mathrm{YbZnGaO}_{4}$ with temperatures spanning about 3 decades, ranging from 0.05 to $4 \mathrm{~K}$. Some of the results are shown in Fig. 14. In both

compounds, they observe strong frequency-dependent peaks below $0.1 \mathrm{~K}$, evidencing a broad distribution of the spin relaxation times around $T_{\mathrm{f}}$, typical for a spin glass [136 142]. They 
(a)

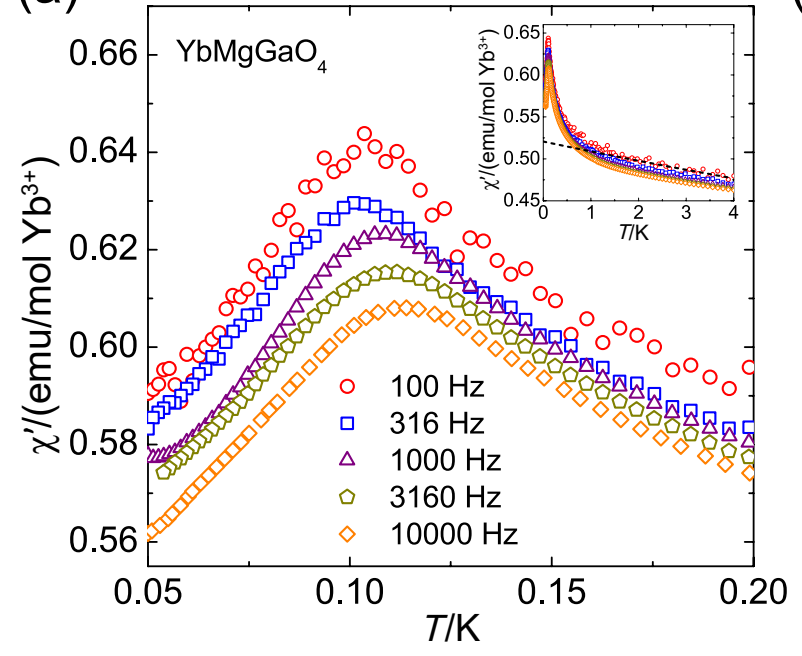

(b)

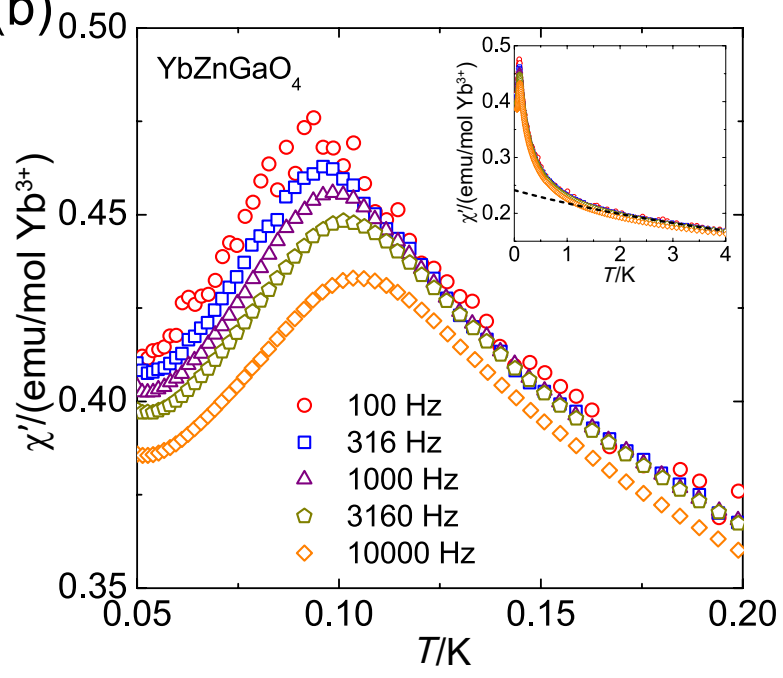

FIG. 14. (a) and (b) Temperature dependence of the a.c. susceptibility $\left(\chi^{\prime}\right)$ for $\mathrm{YbMgGaO}_{4}$ and $\mathrm{YbZnGaO}_{4}$, respectively. In the insets, $\chi^{\prime}$ in an extended temperature range up to $4 \mathrm{~K}$ are plotted. Dashed lines indicate the Curie-Weiss fits for the 100-Hz data. From ref. [51].

consider disorder and frustration to give rise to the spin-glass phase.

However, a $\mu \mathrm{SR}$ study on $\mathrm{YbMgGaO}_{4}$ [47] shows that there is no signature of spin freezing down to $0.07 \mathrm{~K}$, which is already below the $T_{\mathrm{f}}$ reported in the a.c. susceptibility measurements[51]. One possible origin of this discrepancy is that these two techniques cover different time scales: the former and latter probes are sensitive to fluctuations with frequencies larger and smaller than $10^{4} \mathrm{~Hz}$, respectively [143]. Furthermore, as estimated from the INS results, the portions of the spectral weight in the elastic channel over the total weight, are $16 \%$ and $13 \%$ for $\mathrm{YbMgGaO}_{4}$ and $\mathrm{YbZnGaO}_{4}$, respectively [45, 51]. These roughly represent the portions of moments that have been frozen. All these together may cause difficulties to detect the spin freezing by $\mu \mathrm{SR}$.

Another important feature for a spin glass is that in the d.c. susceptibility measurements, there should be a cusp at $T_{\mathrm{f}}$, where the zero-field-cooling and field-cooling susceptibility begin to separate from each other. At present, there are no such data available for $\mathrm{YbMgGaO}_{4}$ and $\mathrm{YbZnGaO}_{4}$, so performing d.c. susceptibility down to temperatures below $0.1 \mathrm{~K}$ will be useful to further clarify the ground state of these compounds.

For organic compounds such as $\kappa$ - $(\mathrm{BEDT}-\mathrm{TTF})_{2} \mathrm{Cu}_{2}(\mathrm{CN})_{3}$ and $\mathrm{EtMe}_{3} \mathrm{Sb}\left[\mathrm{Pd}(\mathrm{dmit})_{2}\right]_{2}$, disorder effect is expected to be significant, and it is unclear that whether the QSL phase 
can survive in the presence of strong disorder[11, 21, 29 32, 144 146]. Moreover, as we discuss in Sec. II B for $\kappa$-(BEDT-TTF $)_{2} \mathrm{Cu}_{2}(\mathrm{CN})_{3}$, the specific heat indicates a gapless ground state. On the other hand, thermal conductivity measurements reveal no contributions from the magnetic excitations, inconsistent with the gapless QSL state. How to reconcile these contradicting results? Is it because that the disorder effect makes the spinons localized and thus not conduct heat? Or the ground state is not a QSL at all? At the current stage, we believe these are open questions calling for further investigations.

For the most heavily studied kagomé compound, $\mathrm{ZnCu}_{3}(\mathrm{OH})_{6} \mathrm{Cl}_{2}$, disorder also plays an important role. In particular, there are 5-15\% excess $\mathrm{Cu}^{2+}$ replacing the nonmagnetic $\mathrm{Zn}^{2+}$, which induces randomness in the magnetic exchange coupling[76, 147, 148]. It is believed that such disorder can be accountable for many experimental observations [76, 84, 147, 149155]. As an example, by considering the $\mathrm{Cu}$ impurities, Han et al. 85] estimate a spin gap of $0.7 \mathrm{meV}$ in the kagomé layer, close to that obtained from the NMR results [80].

For QSL candidates, frustration is strong. In the presence of strong disorder, the spinglass phase is often observed, as disorder and frustration are two important ingredients for a spin glass 136 138, 156]. A spin glass mimics a QSL in many aspects - it maintains shortrange spin-spin correlations, so in the susceptibility, specific heat, and neutron diffraction measurements, it lacks the signature of a long-range magnetic order; moreover, as demonstrated in ref. [51], a spin-glass phase can also produce the continuous INS spectra, which is arguably the strongest evidence for a QSL so far. Therefore, in the quest for QSLs, the spin-glass phase which can give rise to spin-liquid-like features must be excluded first before labeling the candidate as a QSL.

Based on discussions above, we now give several perspectives:

- Although great progress has been made in theory [11, 21, 29 40], it still lacks a proposal for the defining feature of a QSL that can be detected directly from experiments. At present, observations of the continuous magnetic excitation spectra in INS measurements have often been taken to be the most reliable evidence for a QSL[45, 46, 82]. However, this is a necessary but not sufficient evidence for the fractionalized excitations[51]. A feasible direct proposal to identify a QSL should greatly boost this field.

- As we discuss above, there appear to be no ideal QSLs so far. Materials wise, does 
there exist a QSL candidate with large magnetic exchange interactions, little disorder, and minimal extra interactions that produce the static magnetic order? In the past, most attention had been paid to materials with triangular or kagomé lattice where strong geometrical frustration is present[11]. Now, studying the SOC-assisted Mott insulators with anisotropic bond-dependent Kitaev interactions on the honeycomb lattice may offer new possibilities[18, 19, 105]. For instance, very recently, $\mathrm{H}_{3} \mathrm{LiIr}_{2} \mathrm{O}_{6}$ has been suggested to be a Kitaev QSL[157].

- According to Anderson's proposal, high-temperature superconductivity can emerge from QSLs [25 27]. There have been some successes in making QSL candidates superconducting by applying pressures to some organic compounds [33, 61, 69, 158, 159]. However, another more common route to achieve superconductivity — via chemical doping, has not been successful so far[160]. Is it because there is no ideal QSL candidate so far? Will chemical doping an ideal QSL eventually lead to high-temperature superconductivity as predicted? In this aspect, recent advances in doping using electric-field gating may offer some assistance $[161-166]$.

\section{SUMMARY}

To summarize, we review recent progress on QSLs, especially on the magnetic-field measurements on several QSL candidates, including the geometrically-frustrated triangular and kagomé compounds, including $\mathrm{YbMgGaO}_{4}, \mathrm{YbZnGaO}_{4}, \kappa-(\mathrm{ET})_{2} \mathrm{Cu}_{2}(\mathrm{CN})_{3}$, $\mathrm{EtMe}_{3} \mathrm{Sb}\left[\mathrm{Pd}(\mathrm{dmit})_{2}\right]_{2}$, and $\mathrm{ZnCu}_{3}(\mathrm{OH})_{6} \mathrm{Cl}_{2}$, and the Kitaev material $\alpha-\mathrm{RuCl}_{3}$ with the honeycomb lattice. While there are many experimental evidences showing that they are promising candidates for QSLs, there are also some evidences that may be used to argue against the QSL picture. As such, we provide several perspectives hoping to stimulate further investigations. We anticipate that continuous efforts will be paid off by the discovery of more fascinating physics and ideal candidate materials.

Before ending this review, we note that there are many other materials that have been proposed to be QSLs. We list a few examples below:

- $\mathrm{Na}_{4} \mathrm{Ir}_{3} \mathrm{O}_{8}$ is a widely studied QSL candidate with the hyperkagomé lattice[167-170]. In the initial report [167] that suggested it to a QSL, spin freezing indicative of a spin- 
glass phase at $T_{\mathrm{f}}=6 \mathrm{~K}$ was observed. The frozen moments were estimated to be less than $10 \%$ of the total moments and were thus ignored. Later on, both $\mu \mathrm{SR}[169]$ and NMR measurements[171] showed that the spins are frozen and maintain short-range correlations in the ground state.

- Kagomé compounds $\mathrm{ZnCu}_{3}(\mathrm{OH})_{6} \mathrm{SO}_{4}[172]$ and $\mathrm{Zn}$-substituted barlowite $\mathrm{Cu}_{3} \mathrm{Zn}(\mathrm{OH})_{6} \mathrm{FBr}[173$, 174], and a hyperkagomé material $\mathrm{PbCuTe}_{2} \mathrm{O}_{6}\left[175\right.$, 176]. In $\mathrm{Cu}_{3} \mathrm{Zn}(\mathrm{OH})_{6} \mathrm{FBr}$, it has been shown that the magnetic field dependence of the gap extracted from the NMR data is consistent with that given by fractionalized spin-1/2 spinon excitations [173].

- A triangular spin-1 material $\mathrm{Ba}_{3} \mathrm{NiSb}_{2} \mathrm{O}_{9}[177]$.

- $\mathrm{Ca}_{10} \mathrm{Cr}_{7} \mathrm{O}_{28}$, a system with complex structure, and more interestingly, with ferromagnetic interactions [178 [180]. In this compound, although Balz et al. found that there is no static magnetic order in the $\mu \mathrm{SR}$ measurements, they observed frequency dependent peaks in the a.c. susceptibility, which is characteristic of a spin glass [178. However, they argued that the spin-glass phase could be ruled out by doing the ColeCole analysis for the a.c. susceptibility data[178].

- Very recently, a protypical charge-density-wave compound $1 \mathrm{~T}-\mathrm{TaS}_{2}$ with the Davidstar structure has attracted a lot of attention due the possibility of realizing the QSL state[181[185].

In this review, we do not discuss these materials in details due to the limited space. Readers who are interested in them can refer to the above references and the references therein.

\section{ACKNOWLEDGEMENTS}

The work was supported by the National Natural Science Foundation of China with Grant Nos 11674157 and 11822405, and Fundamental Research Funds for the Central Universities with Grant No. 020414380105. We would like to thank our colleagues and collaborators Jian-Xin Li, Shun-Li Yu, Jun-Ming Liu, Shiyan Li, Weiqiang Yu, Xin Lu, P. Čermák, A. Schneidewind, Guochu Deng, S. Danilkin, S. Yano, and J. S. Gardner, who made this 
work possible.

[1] Étienne D T d L, Gignoux D and Schlenker M 2005 Magnetism: Fundamentals (Berlin: Springer)

[2] White R M 2007 Quantum Theory of Magnetism: Magnetic Properties of Materials (Berlin: Springer)

[3] Néel L 1971 Science 174985

[4] Anderson P W 1959 Phys. Rev. 1152

[5] White S R and Chernyshev A L 2007 Phys. Rev. Lett. 99127004

[6] Capriotti L, Trumper A E and Sorella S 1999 Phys. Rev. Lett. 823899

[7] Singh R R P and Huse D A 1992 Phys. Rev. Lett. 681766

[8] Bernu B, Lecheminant P, Lhuillier C and Pierre L 1994 Phys. Rev. B 5010048

[9] Bernu B, Lhuillier C and Pierre L 1992 Phys. Rev. Lett. 692590

[10] Pomeranchuk I Y 1941 Zh. Eksp. Teor. Fiz. 11226

[11] Balents L 2010 Nature 464199

[12] Lee P A 2008 Science 3211306

[13] Wen X G 2002 Phys. Rev. B 65165113

[14] Anderson P 1973 Mater. Res. Bull. 8153

[15] Moessner R and Sondhi S L 2002 Progress of Theoretical Physics Supplement 14537

[16] Liang S, Doucot B and Anderson P W 1988 Phys. Rev. Lett. 61365

[17] Fazekas P and Anderson P W 1974 Philosophical Magazine 30423

[18] Kitaev A 2006 Annals of Physics 3212

[19] Jackeli G and Khaliullin G 2009 Phys. Rev. Lett. 102017205

[20] Kitaev A Y 2003 Ann. Phys. 3032

[21] Savary L and Balents L 2017 Reports on Progress in Physics 80016502

[22] Nayak C, Simon S H, Stern A, Freedman M and Das Sarma S 2008 Rev. Mod. Phys. 801083

[23] Barkeshli M, Berg E and Kivelson S 2014 Science 346722

[24] Bednorz J G and Müller K A 1986 Z. Phys. B 64189

[25] Anderson P W 1987 Science 2351196

[26] Anderson P W, Baskaran G, Zou Z and Hsu T 1987 Phys. Rev. Lett. 582790 
[27] Baskaran G, Zou Z and Anderson P 1987 Solid State Communications 63973

[28] Kivelson S A, Rokhsar D S and Sethna J P 1987 Phys. Rev. B 35 8865(R)

[29] Kanoda K and Kato R 2011 Annual Review of Condensed Matter Physics 2167

[30] Witczak-Krempa W, Chen G, Kim Y B and Balents L 2014 Ann. Rev. Condens. Matter Phys. 557

[31] Zhou Y, Kanoda K and Ng T K 2017 Rev. Mod. Phys. 89025003

[32] Starykh O A 2015 Reports on Progress in Physics 78052502

[33] Powell B J and McKenzie R H 2011 Reports on Progress in Physics 74056501

[34] Kaplan T A and Menyuk N 2007 Philosophical Magazine 873711

[35] Norman M R 2016 Rev. Mod. Phys. 88041002

[36] Rau J G, Lee E K H and Kee H Y 2016 Ann. Rev. Condens. Matter Phys. 7195

[37] Gardner J S, Gingras M J P and Greedan J E 2010 Rev. Mod. Phys. 8253

[38] Castelnovo C, Moessner R and Sondhi S L 2012 Annual Review of Condensed Matter Physics 335

[39] Mendels P and Bert F 2011 Journal of Physics: Conference Series 320012004

[40] Mendels P and Bert F 2010 Journal of the Physical Society of Japan 79011001

[41] Imai T and Lee Y S 2016 Physics Today 6930

[42] Winter S M, Tsirlin A A, Daghofer M, van den Brink J, Singh Y, Gegenwart P and Valent R 2017 Journal of Physics: Condensed Matter 29493002

[43] Li Y, Liao H, Zhang Z, Li S, Jin F, Ling L, Zhang L, Zou Y, Pi L, Yang Z, Wang J, Wu Z and Zhang Q 2015 Sci. Rep. 516419

[44] Li Y, Chen G, Tong W, Pi L, Liu J, Yang Z, Wang X and Zhang Q 2015 Phys. Rev. Lett. 115167203

[45] Paddison J A M, Daum M, Dun Z, Ehlers G, Liu Y, Stone M B, Zhou H and Mourigal M 2017 Nature Phys. 13117

[46] Shen Y, Li Y D, Wo H, Li Y, Shen S, Pan B, Wang Q, Walker H C, Steffens P, Boehm M, Hao Y, Quintero-Castro D L, Harriger L W, Frontzek M D, Hao L, Meng S, Zhang Q, Chen G and Zhao J 2016 Nature $\mathbf{5 4 0} 559$

[47] Li Y, Adroja D, Biswas P K, Baker P J, Zhang Q, Liu J, Tsirlin A A, Gegenwart P and Zhang Q 2016 Phys. Rev. Lett. 117097201 
[48] Li Y, Adroja D, Bewley R I, Voneshen D, Tsirlin A A, Gegenwart P and Zhang Q 2017 Phys. Rev. Lett. 118107202

[49] Li Y, Adroja D, Voneshen D, Bewley R I, Zhang Q, Tsirlin A A and Gegenwart P 2017 Nature Commun. 815814

[50] Xu Y, Zhang J, Li Y S, Yu Y J, Hong X C, Zhang Q M and Li S Y 2016 Phys. Rev. Lett. 117267202

[51] Ma Z, Wang J, Dong Z Y, Zhang J, Li S, Zheng S H, Yu Y, Wang W, Che L, Ran K, Bao S, Cai Z, Čermák P, Schneidewind A, Yano S, Gardner J S, Lu X, Yu S L, Liu J M, Li S, Li J X and Wen J 2018 Phys. Rev. Lett. 120087201

[52] Balents L 2016 Nature 540534

[53] Zhu Z, Maksimov P A, White S R and Chernyshev A L 2017 Phys. Rev. Lett. 119157201

[54] Yamashita M, Nakata N, Senshu Y, Nagata M, Yamamoto H M, Kato R, Shibauchi T and Matsuda Y 2010 Science $\mathbf{3 2 8} 1246$

[55] Nave C P and Lee P A 2007 Phys. Rev. B 76235124

[56] Motrunich O I 2005 Phys. Rev. B 72045105

[57] Kino H and Fukuyama H 1995 Journal of the Physical Society of Japan 642726

[58] Shimizu Y, Miyagawa K, Kanoda K, Maesato M and Saito G 2005 Progress of Theoretical Physics Supplement 15952

[59] Komatsu T, Matsukawa N, Inoue T and Saito G 1996 Journal of the Physical Society of Japan 651340

[60] Shimizu Y, Miyagawa K, Kanoda K, Maesato M and Saito G 2003 Phys. Rev. Lett. 91107001

[61] Kurosaki Y, Shimizu Y, Miyagawa K, Kanoda K and Saito G 2005 Phys. Rev. Lett. 95 177001

[62] Ohira S, Shimizu Y, Kanoda K and Saito G 2006 Journal of Low Temperature Physics 142 153

[63] Furukawa T, Kobashi K, Kurosaki Y, Miyagawa K and Kanoda K 2018 Nature Communications $\mathbf{9} 307$

[64] Miyagawa K, Kanoda K and Kawamoto A 2004 Chemical Reviews 1045635

[65] Pratt F L, Baker P J, Blundell S J, Lancaster T, Ohira-Kawamura S, Baines C, Shimizu Y, Kanoda K, Watanabe I and Saito G 2011 Nature 471612 
[66] Yamashita S, Nakazawa Y, Oguni M, Oshima Y, Nojiri H, Shimizu Y, Miyagawa K and Kanoda K 2008 Nat Phys 4459

[67] Yamashita M, Nakata N, Kasahara Y, Sasaki T, Yoneyama N, Kobayashi N, Fujimoto S, Shibauchi T and Matsuda Y 2009 Nat Phys 544

[68] Kato R 2014 Bulletin of the Chemical Society of Japan 87355

[69] Shimizu Y, Akimoto H, Tsujii H, Tajima A and Kato R 2007 Phys. Rev. Lett. 99256403

[70] Itou T, Oyamada A, Maegawa S and Kato R 2010 Nature Physics 6673

[71] Yamashita S, Yamamoto T, Nakazawa Y, Tamura M and Kato R 2011 Nature Communications 2275

[72] Shimizu Y, Akimoto H, Tsujii H, Tajima A and Kato R 2007 Journal of Physics Condensed Matter 19145240

[73] Tamura M, Nakao A and Kato R 2006 Journal of the Physical Society of Japan 75093701

[74] Nytko E A, Helton J S, Mller P and Nocera D G 2008 Journal of the American Chemical Society 1302922

[75] Shores M P, Nytko E A, Bartlett B M, and Nocera D G 2005 Journal of the American Chemical Society $\mathbf{1 2 7} 13462$

[76] Han T H, Helton J S, Chu S, Prodi A, Singh D K, Mazzoli C, Müller P, Nocera D G and Lee Y S 2011 Phys. Rev. B 83100402

[77] Helton J S, Matan K, Shores M P, Nytko E A, Bartlett B M, Yoshida Y, Takano Y, Suslov A, Qiu Y, Chung J H, Nocera D G and Lee Y S 2007 Phys. Rev. Lett. 98107204

[78] Mendels P, Bert F, de Vries M A, Olariu A, Harrison A, Duc F, Trombe J C, Lord J S, Amato A and Baines C 2007 Phys. Rev. Lett. 98077204

[79] de Vries M A, Stewart J R, Deen P P, Piatek J O, Nilsen G J, Rønnow H M and Harrison A 2009 Phys. Rev. Lett. 103237201

[80] Fu M, Imai T, Han T H and Lee Y S 2015 Science 350655

[81] Jeong M, Bert F, Mendels P, Duc F, Trombe J C, de Vries M A and Harrison A 2011 Phys. Rev. Lett. 107237201

[82] Han T H, Helton J S, Chu S, Nocera D G, Rodriguez-Rivera J A, Broholm C and Lee Y S 2012 Nature 492406

[83] Yan S, Huse D A and White S R 2011 Science 3321173 
[84] Asaba T, Han T H, Lawson B J, Yu F, Tinsman C, Xiang Z, Li G, Lee Y S and Li L 2014 Phys. Rev. B 90064417

[85] Han T H, Norman M R, Wen J J, Rodriguez-Rivera J A, Helton J S, Broholm C and Lee Y S 2016 Phys. Rev. B 94060409

[86] Liao H J, Xie Z Y, Chen J, Liu Z Y, Xie H D, Huang R Z, Normand B and Xiang T 2017 Phys. Rev. Lett. 118137202

[87] Chaloupka J, Jackeli G and Khaliullin G 2010 Phys. Rev. Lett. 105027204

[88] Shitade A, Katsura H, Kuneš J, Qi X L, Zhang S C and Nagaosa N 2009 Phys. Rev. Lett. 102256403

[89] Chaloupka J, Jackeli G and Khaliullin G 2013 Phys. Rev. Lett. 110097204

[90] Yamaji Y, Suzuki T, Yamada T, Suga S i, Kawashima N and Imada M 2016 Phys. Rev. B 93174425

[91] Mehlawat K, Thamizhavel A and Singh Y 2017 Phys. Rev. B 95144406

[92] Modic K A, Smidt T E, Kimchi I, Breznay N P, Biffin A, Choi S, Johnson R D, Coldea R, Watkins-Curry P, McCandless G T, Chan J Y, Gandara F, Islam Z, Vishwanath A, Shekhter A, McDonald R D and Analytis J G 2014 Nature Communications 54203

[93] Takayama T, Kato A, Dinnebier R, Nuss J, Kono H, Veiga L S I, Fabbris G, Haskel D and Takagi H 2015 Phys. Rev. Lett. 114077202

[94] Williams S C, Johnson R D, Freund F, Choi S, Jesche A, Kimchi I, Manni S, Bombardi A, Manuel P, Gegenwart P and Coldea R 2016 Phys. Rev. B 93195158

[95] Glamazda A, Lemmens P, Do S H, Choi Y S and Choi K Y 2016 Nature Communications 7 12286

[96] Gretarsson H, Clancy J P, Liu X, Hill J P, Bozin E, Singh Y, Manni S, Gegenwart P, Kim J, Said A H, Casa D, Gog T, Upton M H, Kim H S, Yu J, Katukuri V M, Hozoi L, van den Brink J and Kim Y J 2013 Phys. Rev. Lett. 110076402

[97] Comin R, Levy G, Ludbrook B, Zhu Z H, Veenstra C N, Rosen J A, Singh Y, Gegenwart P, Stricker D, Hancock J N, van der Marel D, Elfimov I S and Damascelli A 2012 Phys. Rev. Lett. 109266406

[98] Singh Y and Gegenwart P 2010 Phys. Rev. B 82064412

[99] Liu X, Berlijn T, Yin W G, Ku W, Tsvelik A, Kim Y J, Gretarsson H, Singh Y, Gegenwart P and Hill J P 2011 Phys. Rev. B 83220403 
[100] Choi S K, Coldea R, Kolmogorov A N, Lancaster T, Mazin I I, Blundell S J, Radaelli P G, Singh Y, Gegenwart P, Choi K R, Cheong S W, Baker P J, Stock C and Taylor J 2012 Phys. Rev. Lett. 108127204

[101] Ye F, Chi S, Cao H, Chakoumakos B C, Fernandez-Baca J A, Custelcean R, Qi T F, Korneta O B and Cao G 2012 Phys. Rev. B 85180403

[102] Singh Y, Manni S, Reuther J, Berlijn T, Thomale R, Ku W, Trebst S and Gegenwart P 2012 Phys. Rev. Lett. 108127203

[103] Fletcher J M, Gardner W E, Hooper E W, Hyde K R, Moore F H and Woodhead J L 1963 Nature 1991089

[104] Fletcher J M, Gardner W E, Fox A C and Topping G 1967 J. Chem. Soc. A 1038

[105] Plumb K W, Clancy J P, Sandilands L J, Shankar V V, Hu Y F, Burch K S, Kee H Y and Kim Y J 2014 Phys. Rev. B 90041112

[106] Kubota Y, Tanaka H, Ono T, Narumi Y and Kindo K 2015 Phys. Rev. B 91094422

[107] Sears J A, Songvilay M, Plumb K W, Clancy J P, Qiu Y, Zhao Y, Parshall D and Kim Y J 2015 Phys. Rev. B 91144420

[108] Cao H B, Banerjee A, Yan J Q, Bridges C A, Lumsden M D, Mandrus D G, Tennant D A, Chakoumakos B C and Nagler S E 2016 Phys. Rev. B 93134423

[109] Guizzetti G, Reguzzoni E and Pollini I 1979 Physics Letters A 7034

[110] Binotto L, Pollini I and Spinolo G 1971 physica status solidi (b) 44245

[111] Pollini I 1996 Phys. Rev. B 5312769

[112] Pollini I 1994 Phys. Rev. B 502095

[113] Hou Y S, Xiang H J and Gong X G 2017 Phys. Rev. B 96054410

[114] Banerjee A, Bridges C, Yan J, Aczel A, Li L, Stone M, Granroth G, Lumsden M, Yiu Y, Knolle J et al. 2016 Nature Mat. 15733

[115] Ran K, Wang J, Wang W, Dong Z Y, Ren X, Bao S, Li S, Ma Z, Gan Y, Zhang Y, Park J T, Deng G, Danilkin S, Yu S L, Li J X and Wen J 2017 Phys. Rev. Lett. 118107203

[116] Banerjee A, Yan J, Knolle J, Bridges C A, Stone M B, Lumsden M D, Mandrus D G, Tennant D A, Moessner R and Nagler S E 2017 Science 3561055

[117] Do S H, Park S Y, Yoshitake J, Nasu J, Motome Y, Kwon Y S, Adroja D T, Voneshen D J, Kim K, Jang T H, Park J H, Choi K Y and Ji S 2017 Nature Phys. 131079 
[118] Sandilands L J, Tian Y, Plumb K W, Kim Y J and Burch K S 2015 Phys. Rev. Lett. 114 147201

[119] Nasu J, Knolle J, Kovrizhin D L, Motome Y and Moessner R 2016 Nature Phys. 12912

[120] Wang W, Dong Z Y, Yu S L and Li J X 2017 Phys. Rev. B 96115103

[121] Majumder M, Schmidt M, Rosner H, Tsirlin A A, Yasuoka H and Baenitz M 2015 Phys. Rev. B 91180401

[122] Johnson R D, Williams S C, Haghighirad A A, Singleton J, Zapf V, Manuel P, Mazin I I, Li Y, Jeschke H O, Valentí R and Coldea R 2015 Phys. Rev. B 92235119

[123] Cui Y, Zheng J, Ran K, Wen J, Liu Z X, Liu B, Guo W and Yu W 2017 Phys. Rev. B 96 205147

[124] Zheng J, Ran K, Li T, Wang J, Wang P, Liu B, Liu Z X, Normand B, Wen J and Yu W 2017 Phys. Rev. Lett. 119227208

[125] Wolter A U B, Corredor L T, Janssen L, Nenkov K, Schönecker S, Do S H, Choi K Y, Albrecht R, Hunger J, Doert T, Vojta M and Büchner B 2017 Phys. Rev. B 96041405

[126] Sears J A, Zhao Y, Xu Z, Lynn J W and Kim Y J 2017 Phys. Rev. B 95180411

[127] Leahy I A, Pocs C A, Siegfried P E, Graf D, Do S H, Choi K Y, Normand B and Lee M 2017 Phys. Rev. Lett. 118187203

[128] Aoyama T, Hasegawa Y, Kimura S, Kimura T and Ohgushi K 2017 Phys. Rev. B 95245104

[129] Little A, Wu L, Lampen-Kelley P, Banerjee A, Patankar S, Rees D, Bridges C A, Yan J Q, Mandrus D, Nagler S E and Orenstein J 2017 Phys. Rev. Lett. 119227201

[130] Wang Z, Reschke S, Hüvonen D, Do S H, Choi K Y, Gensch M, Nagel U, Rõ om T and Loidl A 2017 Phys. Rev. Lett. 119227202

[131] Ponomaryov A N, Schulze E, Wosnitza J, Lampen-Kelley P, Banerjee A, Yan J Q, Bridges C A, Mandrus D G, Nagler S E, Kolezhuk A K and Zvyagin S A 2017 Phys. Rev. B 96 241107

[132] Banerjee A, Lampen-Kelley P, Knolle J, Balz C, Aczel A A, Winn B, Liu Y, Pajerowski D, Yan J, Bridges C A, Savici A T, Chakoumakos B C, Lumsden M D, Tennant D A, Moessner R, Mandrus D G and Nagler S E 2018 npj Quantum Materials 38

[133] Hentrich R, Wolter A U B, Zotos X, Brenig W, Nowak D, Isaeva A, Doert T, Banerjee A, Lampen-Kelley P, Mandrus D G, Nagler S E, Sears J, Kim Y J, Büchner B and Hess C 2018 Phys. Rev. Lett. 120117204 
[134] Baek S H, Do S H, Choi K Y, Kwon Y S, Wolter A U B, Nishimoto S, van den Brink J and Büchner B 2017 Phys. Rev. Lett. 119037201

[135] Yu Y J, Xu Y, Ran K J, Ni J M, Huang Y Y, Wang J H, Wen J S and Li S Y 2018 Phys. Rev. Lett. 120067202

[136] Binder K and Young A P 1986 Rev. Mod. Phys. 58801

[137] Mydosh J A 1993 Spin glasses : an experimental introduction (London: Taylor \& Francis)

[138] Mydosh J A 1986 Hyperfine Interactions 31347

[139] Hüser D, Wenger L E, van Duyneveldt A J and Mydosh J A 1983 Phys. Rev. B 273100

[140] Mulder C A M, van Duyneveldt A J and Mydosh J A 1981 Phys. Rev. B 231384

[141] Eiselt G, Kötzler J, Maletta H, Stauffer D and Binder K 1979 Phys. Rev. B 192664

[142] Mehlawat K, Sharma G and Singh Y 2015 Phys. Rev. B 92134412

[143] Sonier J E 2002 http://phys.columbia.edu/fcmp/files//FCMP2015_MuSR_Brochure.pdf

[144] Watanabe K, Kawamura H, Nakano H and Sakai T 2014 Journal of the Physical Society of Japan 83034714

[145] Kawamura H, Watanabe K and Shimokawa T 2014 Journal of the Physical Society of Japan 83103704

[146] Shimokawa T, Watanabe K and Kawamura H 2015 Phys. Rev. B 92134407

[147] Freedman D E, Han T H, Prodi A, Mller P, Huang Q Z, Chen Y S, Webb S M, Lee Y S, McQueen T M and Nocera D G 2010 Journal of the American Chemical Society 13216185

[148] Han T, Chu S and Lee Y S 2012 Phys. Rev. Lett. 108157202

[149] Olariu A, Mendels P, Bert F, Duc F, Trombe J C, de Vries M A and Harrison A 2008 Phys. Rev. Lett. 100087202

[150] Chitra R and Rozenberg M J 2008 Phys. Rev. B 77052407

[151] Imai T, Nytko E A, Bartlett B M, Shores M P and Nocera D G 2008 Phys. Rev. Lett. 100 077203

[152] de Vries M A, Kamenev K V, Kockelmann W A, Sanchez-Benitez J and Harrison A 2008 Phys. Rev. Lett. 100157205

[153] Bert F, Nakamae S, Ladieu F, L'Hôte D, Bonville P, Duc F, Trombe J C and Mendels P 2007 Phys. Rev. B $\mathbf{7 6} 132411$

[154] Lee S H, Kikuchi H, Qiu Y, Lake B, Huang Q, Habicht K and Kiefer K 2007 Nat Mater 6 853 
[155] Rozenberg M J and Chitra R 2008 Phys. Rev. B 78132406

[156] Wu J, Wildeboer J S, Werner F, Seidel A, Nussinov Z and Solin S A 2011 Euro Phys. Lett. 9367001

[157] Kitagawa K, Takayama T, Matsumoto Y, Kato A, Takano R, Kishimoto Y, Bette S, Dinnebier R, Jackeli G and Takagi H 2018 Nature 554341

[158] Lefebvre S, Wzietek P, Brown S, Bourbonnais C, Jérome D, Mézière C, Fourmigué M and Batail P 2000 Phys. Rev. Lett. 855420

[159] Kato R, Tajima A, Nakao A and Tamura M 2006 Journal of the American Chemical Society 12810016

[160] Kelly Z A, Gallagher M J and McQueen T M 2016 Phys. Rev. X 6041007

[161] Ueno K, Shimotani H, Yuan H, Ye J, Kawasaki M and Iwasa Y 2014 Journal of the Physical Society of Japan $\mathbf{8 3} 032001$

[162] Lei B, Cui J H, Xiang Z J, Shang C, Wang N Z, Ye G J, Luo X G, Wu T, Sun Z and Chen X H 2016 Phys. Rev. Lett. 116077002

[163] Bollinger A T, Dubuis G, Yoon J, Pavuna D, Misewich J and Božović I 2011 Nature 472 458

[164] Lu N, Zhang P, Zhang Q, Qiao R, He Q, Li H B, Wang Y, Guo J, Zhang D, Duan Z, Li Z, Wang M, Yang S, Yan M, Arenholz E, Zhou S, Yang W, Gu L, Nan C W, Wu J, Tokura Y and Yu P 2017 Nature 546124

[165] Cui Y, Zhang G, Li H, Lin H, Zhu X, Wen H H, Wang G, Sun J, Ma M, Li Y, Gong D, Xie T, Gu Y, Li S, Luo H, Yu P and Yu W 2018 Science Bulletin 6311

[166] Hosono H 2018 Science Bulletin 635

[167] Okamoto Y, Nohara M, Aruga-Katori H and Takagi H 2007 Phys. Rev. Lett. 99137207

[168] Singh Y, Tokiwa Y, Dong J and Gegenwart P 2013 Phys. Rev. B 88220413

[169] Dally R, Hogan T, Amato A, Luetkens H, Baines C, Rodriguez-Rivera J, Graf M J and Wilson S D 2014 Phys. Rev. Lett. 113247601

[170] Rehn J, Sen A and Moessner R 2017 Phys. Rev. Lett. 118047201

[171] Shockley A C, Bert F, Orain J C, Okamoto Y and Mendels P 2015 Phys. Rev. Lett. 115 047201

[172] Li Y, Pan B, Li S, Tong W, Ling L, Yang Z, Wang J, Chen Z, Wu Z and Zhang Q 2014 New Journal of Physics 16093011 
[173] Feng Z, Li Z, Meng X, Yi W, Wei Y, Zhang J, Wang Y C, Jiang W, Liu Z and Li S 2017 Chinese Physics Letters 347

[174] Wen X G 2017 Chinese Physics Letters 34090101

[175] Koteswararao B, Kumar R, Khuntia P, Bhowal S, Panda S K, Rahman M R, Mahajan A V, Dasgupta I, Baenitz M, Kim K H and Chou F C 2014 Physical Review B 90035141

[176] Khuntia P, Bert F, Mendels P, Koteswararao B, Mahajan A V, Baenitz M, Chou F C, Baines C, Amato A and Furukawa Y 2016 Phys. Rev. Lett. 116107203

[177] Fåk B, Bieri S, Canévet E, Messio L, Payen C, Viaud M, Guillot-Deudon C, Darie C, Ollivier J and Mendels P 2017 Phys. Rev. B 95060402

[178] Balz C, Lake B, Reuther J, Luetkens H, Schonemann R, Herrmannsdorfer T, Singh Y, Nazmul Islam A T M, Wheeler E M, Rodriguez-Rivera J A, Guidi T, Simeoni G G, Baines C and Ryll H 2016 Nature Phys. 12942

[179] Balz C, Lake B, Nazmul Islam A T M, Singh Y, Rodriguez-Rivera J A, Guidi T, Wheeler E M, Simeoni G G and Ryll H 2017 Phys. Rev. B 95174414

[180] Balz C, Lake B, Reehuis M, Islam A T M N, Prokhnenko O, Singh Y, Pattison P and Tth S 2017 Journal of Physics: Condensed Matter 29225802

[181] Law K T and Lee P A 2017 Proceedings of the National Academy of Sciences 1146996

[182] Kratochvilova M, Hillier A D, Wildes A R, Wang L, Cheong S W and Park J G $2017 n p j$ Quantum Materials 242

[183] Klanjšek M, Zorko A, Žitko R, Mravlje J, Jagličić Z, Biswas P K, Prelovšek P, Mihailovic D and Arčon D 2017 Nature Phys. 131130

[184] Ribak A, Silber I, Baines C, Chashka K, Salman Z, Dagan Y and Kanigel A 2017 Phys. Rev. B 96195131

[185] Yu Y J, Xu Y, He L P, Kratochvilova M, Huang Y Y, Ni J M, Wang L, Cheong S W, Park J G and Li S Y 2017 Phys. Rev. B 96081111 